\title{
HARMONIC BUNDLES ON NONCOMPACT CURVES
}

\author{
CARLOS T. SIMPSON
}

\section{CONTENTS}

Preface

Synopsis

1. Harmonic bundles

2. Main estimate

3. Filtered objects

4. Sections and morphisms

5. Local study

6. Stability and existence results

7. Weight filtrations

8. Variations of Hodge structure

References

\section{Preface}

The purpose of this paper is to extend the correspondence between Higgs bundles and local systems $[2,5,6,7,13,17,19,20,21]$ to the case when $X$ is a noncompact algebraic curve. The basic result is that there is a class of analytic objects on $X$ which will be put in one-to-one correspondences with two different classes of algebraic geometric objects on the completion $\bar{X}$. The analytic objects are harmonic bundles on $X$ satisfying a growth condition at the punctures which we call tameness. The two types of algebraic objects are Higgs bundles and $\mathscr{D}_{X}$-modules, both with regular singularities at the punctures, together with additional data of filtrations of the fibers over the punctures. The filtered regular $\mathscr{D}_{X}$-modules also correspond to topological objects, local systems (i.e. representations of the fundamental group), together with filtrations assigned to the punctures. For the algebraic or topological objects, there is an

Received by the editors January 10, 1990.

1980 Mathematics Subject Classification (1985 Revision). Primary 58E20; Secondary 14E20, 30 F99.

This work was prepared with the partial support of NSF grant DMS-8705757. 
extra condition of stability needed for inclusion in our correspondences. We get one-to-one correspondences between irreducible tame harmonic bundles, stable filtered regular Higgs bundles, stable filtered regular $\mathscr{D}_{X}$-modules, and stable filtered local systems. The reader is referred to the Synopsis for a more detailed description.

The main problem in the compact case was to understand the nonlinear analysis needed to construct analytic objects such as harmonic bundles. However, this being understood by now, we are left in the noncompact case with the problem of figuring out what happens near the singularities at the punctures. In order to simplify the situation, we make what turns out to be a very natural condition of tameness on the growth of the harmonic metric near a puncture. This restriction to tame harmonic bundles allows us to prove a curvature estimate in $\S 2$. The curvature estimate is what gives the algebraic structures across the punctures, as well as the filtrations characterizing growth rates of the harmonic metrics $(\S 3)$. Then in $\S 4$, some Weitzenböck formulas allow us to sharpen the growth estimates. To go the other way, from a filtered algebraic object to a class of metrics, some models are needed for the singularities ( $\$ 5)$. After all of this is done, the machinery from the compact case can take over, with a stability condition being the key to existence of harmonic metrics ( $\S 6)$. Finally, and this is a rather difficult point, we show that the local pictures of the various types of algebraic objects correspond nicely under our maps ( $\S 7)$. In $\S 8$ we give a short application characterizing variations of Hodge structure as fixed points of a natural $\mathbf{C}^{*}$ action.

This paper represents the next logical step after [19]. We are in effect carrying our the program described in the introduction to that paper, for the case of noncompact curves. In fact, the case of variations of Hodge structure on a noncompact curve was already treated there, so some of the steps we need here have already been completed. In these cases we can quote the relevant lemmas.

More generally, the present work builds on what is known in four basic areas. These are the extensions of the theorem of Narasimhan and Seshadri, the theory of harmonic maps, the study of variations of Hodge structure, and the theory of differential equations with regular singularities.

The theorem of Narasimhan and Seshadri [17], relating unitary local systems and stable vector bundles, has been generalized by Donaldson [5, 6], Uhlenbeck and Yau [21], Hitchin [13], Deligne and Beilinson, and the author [19, 20]. The later generalizations introduced an interaction term which Hitchin called the Higgs field, which results in nonunitary connections. In another direction, Mehta and Seshadri worked out the case of unitary connections on a noncompact curve, introducing the idea of filtrations or parabolic structures controlling the singularities of the connection [16]. Most of our analytic difficulties stem from trying to deal with Hitchin's Higgs field in the noncompact case.

The converse problem of going from a local system to a vector bundle or Higgs bundle is trivial in the unitary case, but in the case of nonunitary local systems it involves the notion of equivariant harmonic maps. This definition and the 
main existence theorem are due to Corlette [2] (and Donaldson, independently, for rank 2 [7]), following the original ideas of Eells and Sampson [8] (cf. also [12]). This work in the compact case can be carried over to the noncompact case. In fact, and this is a gap in the exposition, we have not included the proof, but it is by now fairly straightforward.

One of the main sources of inspiration for the methods of this paper has been the theory of variations of Hodge structure, invented by Griffiths [9] and further developed by Schmid [11, 18] (and of course many others). The relation with harmonic bundles is that the variations of Hodge structure provide the most basic examples which demonstrate how to go beyond the unitary case. Griffiths' period map is essentially the same as the equivariant harmonic map-it is a lifting. Schmid studied very carefully the asymptotic behavior of the period map near a singularity. The results point to what to expect of a harmonic map, or at least of a tame one. Schmid's calculation of the curvature of the period domain, and the resulting distance decreasing property [11], are replicated in our main curvature estimate. The models for the behavior of variations of Hodge structure provided by Schmid's $S l_{2}$ orbit theorem [18] provide the models we need to study the local behavior in the tame harmonic case. In using the curvature estimate to gain control of the local picture, we do not follow Schmid's nilpotent orbit and $\mathrm{Sl}_{2}$ orbit theorems. Rather we follow a program started by Griffiths where he proved the regularity of the Gauss-Manin connection (cf. [10]). We use results of Cornalba and Griffiths to extend the algebraic structures of the bundles across the puncture [3]. By going this route, we obtain an alternate proof of the crudest of Schmid's norm estimates, but not the more refined asymptotic information.

The last of the ingredients is the classical theory of differential equations with regular singularities, as formalized in modern terms by Deligne [4] and subsequently pursued by many others under the rubric of $\mathscr{D}_{X}$-modules. Our notion of tameness of a harmonic bundle is closely related to regular singularity of a system of differential equations, and hence, more abstrusely, to the notion of tameness of an $l$-adic representation in number theory (thus our terminology). In fact, tameness of the harmonic bundle leads to regular singularity of the algebraic connection which we construct. We follow ideas of Kashiwara [14] and Malgrange [15] on introducing filtrations at the singularities of a $\mathscr{D}_{X}$-module (as well as the work of Mehta and Seshadri noted above), in making our definitions of filtered objects. The classical correspondence between regular singular systems of differential equations and representations of the fundamental group is very useful in allowing us to interpret our results in terms of topological objects, filtered local systems.

One of the stranger discoveries in the course of this work was the occurrence of filtered local systems as the basic topological objects of study. The additional data of the filtrations correspond to some additional data about the growth rates of the equivariant harmonic map. (It turns out that Deligne had considered filtered local systems with integer jumps. See Appendix C of [23].) 
The possibility of introducing the extra data of filtrations should provide an interesting method to use in the study of the set of representations. Essentially it allows one to construct new representations with different conjugacy classes of monodromy transformations around the punctures-this will be taken up in a later paper.

The following are some of the topics which merit further study. First of all, the restriction of tameness is a fairly strong one, if one is interested in classifying all harmonic bundles. A question is whether one could set up a correspondence in which some nontame harmonic bundles correspond to systems of differential equations with irregular singularities. The main curvature estimate would seem to be more difficult in this case, although that problem could be avoided by looking at bundles of rank 1 first. A second point is that, while we have obtained some norm estimates, we did not generalize Schmid's $S l_{2}$ orbit theorem [18]. This would be an important generalization to make, to obtain higher order asymptotic information on the behavior of the harmonic map. For example, what becomes of the limiting mixed Hodge structure in the harmonic case? Another bit of the theory of variations of Hodge structure which would probably be worth replicating is Zucker's theorem on the $L^{2}$ cohomology with coefficients in a variation of Hodge structure [22]. Some more obvious problems to consider are the problem of constructing nice moduli spaces for local systems in the noncompact case, and of course the problem of treating noncompact varieties in higher dimensions.

I would like to thank W. Schmid, J. Bernstein, K. Corlette, P. Deligne, and J. Simpson.

\section{SYNOPSIS}

This paper contains results about tame harmonic bundles on noncompact curves. $X$ is a smooth noncompact algebraic curve, $\bar{X}$ denotes its smooth completion to a compact Riemann surface, and $S=\bar{X}-X$ is the finite set of punctures. The theme is that there are several types of objects on $X$, two algebraic geometric and one topological, with filtrations at the punctures, which are put in one-to-one correspondence using the notion of tame harmonic bundle. We start by discussing the algebraic and topological objects.

To describe the two kinds of filtered algebraic geometric objects, let us discuss the notion of filtered vector bundle. This is essentially what Mehta and Seshadri called a vector bundle with parabolic structure. An algebraic vector bundle on $X$ is a bundle $E$ given by regular algebraic transition functions over Zariski open sets. We will interchangeably think of $E$ as a locally free sheaf of $\mathscr{O}_{X}$-modules. $E$ may be extended to a locally free sheaf over a puncture $s$ simply by including $s$ in one of the charts. However, the extension will depend on the description of $E$ in terms of transition functions-so there are many ways to extend $E$. The sections of these various extensions are all contained in the set of meromorphic sections of $E$. A filtered vector bundle is an algebraic vector bundle $E$ together with a collection of extensions $E_{\alpha, \dot{s}}$ across the punctures $s$, such that: the 
extensions form a decreasing left continuous filtration: $E_{\alpha, s} \subset E_{\beta, s}$ for $\alpha \geq \beta$, and $E_{\alpha-\varepsilon, s}=E_{\alpha, s}$ for small $\varepsilon$; and if $z$ is a local coordinate vanishing to order one at $s$, then $E_{\alpha+1, s}=z E_{\alpha, s}$.

The filtration is really described by the $\alpha$ between 0 and 1 . Let $\bar{E}$ be the bundle on $\bar{X}$ obtained by using the extensions $E_{0, s}$ at all the punctures. Then the fiber $\bar{E}(s)$ is a vector space with a filtration $\bar{E}_{\alpha}(s)$ indexed by $0 \leq \alpha<1$. The degree of a filtered bundle is defined to be

$$
\operatorname{deg}(E)=\operatorname{deg}(\bar{E})+\sum_{s} \sum_{0 \leq \alpha<1} \alpha \operatorname{dim}\left(\mathrm{Gr}_{\alpha}(\bar{E}(s))\right) .
$$

The main type of algebraic object we will consider is a filtered regular Higgs bundle. This consists of a filtered vector bundle $E$, together with a map

$$
\theta: E \rightarrow E \otimes \Omega_{X}^{1}
$$

of sheaves of $\mathscr{O}_{X}$-modules, satisfying a regularity condition with respect to the filtrations

$$
\theta: E_{\alpha, s} \rightarrow E_{\alpha, s} \otimes \Omega_{\bar{X}}^{1}(\log s) .
$$

Here $\Omega \frac{1}{X}(\log s)$ denotes the sheaf of differentials generated at $s$ by $d z / z$. The degree of $E$ is simply the degree of the filtered bundle. We say that $E$ is stable if, for every subbundle $M \subset E$ (with induced filtration), preserved by $\theta$,

$$
\frac{\operatorname{deg}(M)}{\operatorname{rk}(M)}<\frac{\operatorname{deg}(E)}{\operatorname{rk}(E)}
$$

The objects we will consider will be stable filtered regular Higgs bundles of degree zero.

Our next type of object is the filtered regular $\mathscr{D}_{X}$-module. By this we will mean (and this is an abuse of notation) a filtered vector bundle $V$ together with an algebraic connection

$$
\nabla: V \rightarrow V \otimes \Omega_{X}^{1}
$$

(satisfying the Leibniz rule $\nabla(a v)=d(a) v+a \nabla(v)$, it is automatically integrable), such that it obeys the regularity condition with respect to the filtration

$$
\nabla: V_{\alpha, s} \rightarrow V_{\alpha, s} \otimes \Omega \frac{1}{\bar{X}}(\log s) .
$$

The name $\mathscr{D}_{X}$-module indicates that the connection $\nabla$ gives an action of the sheaf of algebraic differential operators $\mathscr{D}_{X}$ on $V$, although the ones we consider here by no means exhaust all modules over $\mathscr{D}_{X}$.

The degree of a filtered regular $\mathscr{D}_{X}$-module is just the degree of the filtered vector bundle. A $\mathscr{D}_{X}$-module $V$ is stable if, for any subbundle $M \subset V$ (with induced filtration) which is preserved by the connection $\nabla$,

$$
\frac{\operatorname{deg}(M)}{\operatorname{rk}(M)}<\frac{\operatorname{deg}(V)}{\operatorname{rk}(V)} .
$$

The objects we will consider will be stable filtered regular $\mathscr{D}_{X}$-modules of degree zero. 
The regularity condition means in particular that the algebraic connection has regular singularities. There is a classical relation between algebraic connections with regular singularities, and local systems, wherein the local system is the local system of solutions to the system of ordinary differential equations $\nabla(v)=0$. In view of this, we make a definition of topological objects, filtered local systems, which correspond to filtered regular $\mathscr{D}_{X}$-modules. The additional data of the filtration on the local system corresponds to the additional data of the filtration on the $\mathscr{D}_{X}$-module. The correspondence between filtered regular $\mathscr{D}_{X}$-modules and filtered local systems has nothing to do with harmonic bundles, so for the purposes of exposition, one could ignore the $\mathscr{D}_{X}$-modules and state the main theorem as a correspondence between Higgs bundles and local systems.

Here is the notion of filtered local system. A local system is a bundle on $X$ given by constant transition functions. If $x \in X$ is a base point, then a local system $L$ is the same thing as a vector space $L_{x}$ and a representation of $\pi_{1}(X, x)$ on $L_{x}$. Suppose that $s \in S$ is a puncture, and fix a ray $\rho_{s}$ emanating out from $s$. Then it makes sense to discuss the stalk of $L$ over $\rho$, denoted $L_{\rho}$ or, if no confusion arises, $L_{s}$. If $\rho$ is extended to a path back to $x$, then $L_{\rho}$ becomes identified with $L_{x}$. The monodromy transformation "continue once around $s$ " acts on $L_{s}$; denote it by $\mu_{s}$. A filtered local system is a local system $L$ together with filtrations $L_{\beta, s}$ of the stalks $L_{s}$, indexed by real numbers $\beta$. The filtrations are decreasing and left continuous in $\beta$, and $L_{\beta, s}$ should be preserved by the monodromy transformation $\mu_{s}$ (which makes the data of the filtration independent of the choice of $\rho$ ).

The degree of a filtered local system is defined to be

$$
\operatorname{deg}(L)=\sum_{s} \sum_{\beta} \beta \operatorname{dim}\left(\mathrm{Gr}_{\beta}\left(L_{s}\right)\right) .
$$

A filtered local system is said to be stable if for any subsystem $M \subset L$ (with induced filtration),

$$
\frac{\operatorname{deg}(M)}{\operatorname{rk}(M)}<\frac{\operatorname{deg}(L)}{\operatorname{rk}(L)} .
$$

The objects we will consider will be stable filtered local systems of degree zero.

Theorem. There is a natural one-to-one correspondence between stable filtered regular Higgs bundles of degree zero, and stable filtered local systems of degree zero or equivalently stable filtered regular $\mathscr{D}_{x}$-modules of degree zero.

The ranks of the objects are preserved. This correspondence arises from the fact that both sides are shown to correspond to irreducible tame harmonic buna'les.

A harmonic bundle consists of a representation of the fundamental group $\pi_{1}(X, x) \rightarrow \mathrm{Gl}(n, \mathrm{C})$ together with a harmonic map from the universal cover $\tilde{X}$ to the symmetric space $\mathrm{Gl}(n, \mathrm{C}) / \mathrm{U}(n)$, such that the harmonic map is equivariant with respect to the action of the fundamental group on both sides. The harmonic bundle is irreducible if the representation cannot be decomposed as 
a direct sum compatible with the harmonic map. The harmonic map can force irreducibility even if the representation is reducible.

The concept of tameness has to do with the growth of the harmonic map near the punctures. A harmonic bundle is said to be tame if, along any ray out to a puncture, there is a lifting of the harmonic map into a map to $\operatorname{Gl}(n, \mathbf{C})$ which grows at most polynomially in the euclidean distance to the puncture.

An irreducible tame harmonic bundle gives a stable filtered local system of degree zero, and a stable filtered regular Higgs bundle of degree zero. Both of these constructions are one-to-one. The monodromy representation of the local system is the same as the representation in the harmonic bundle, and on the other hand, the $\theta$ of the Higgs bundle essentially gives the derivative of the harmonic map. In both cases, the objects, local systems or Higgs bundles, also come with smoothly varying hermitian metrics defined over $X$. These harmonic metrics satisfy nonlinear partial differential equations. The filtrations measure the growth of the metrics at the punctures.

Important to the usefulness of the correspondence is that there is some control over what happens near the punctures. To describe this, we introduce the notions of residues of the various filtered objects at a puncture $s$. In each of the three cases, the residue is a graded vector space with grading indexed by real numbers which come from the jumps in the filtration, together with an endomorphism which respects the grading.

If $L$ is a filtered local system, then $\operatorname{res}_{s}(L)=\bigoplus_{\beta} \operatorname{Gr}_{\beta}\left(L_{s}\right)$ with endomorphism $\mu_{s}$. Note that this depends on the choice of a ray $\rho$ going from the puncture. If $E$ is a filtered vector bundle, set $\operatorname{res}_{s}(E)=\bigoplus_{0 \leq \alpha<1} \mathrm{Gr}_{\alpha}(\bar{E}(s))$. If $E$ has a map $\theta$ giving a filtered regular Higgs bundle, then $\operatorname{res}_{s}(E)$ is the residue of the filtered bundle defined above, with endomorphism $\operatorname{res}(\theta)$ given by the action of $z \theta(\partial / \partial z)$ on the associated graded. If a filtered vector bundle $V$ has a connection $\nabla$ giving a filtered regular $\mathscr{D}_{X}$-module, then $\operatorname{res}_{s}(V)$ is the residue of the filtered bundle as defined above, with endomorphism $\operatorname{res}(\nabla)$ given by the action of $\nabla(z \partial / \partial z)$ on the associated grading.

One of the main facts is that under our correspondence, the residues of the objects change in a very simple way, up to isomorphism which, however, is not canonical. To describe this, first decompose the residue according to the eigenvalue of the operator. This decomposition is compatible with the grading, so one obtains a decomposition graded by a real number and a complex one. Then on each piece of this finer decomposition, the residual endomorphism acts by a scalar (the eigenvalue) plus a nilpotent. The theorem on equality of the residues is that if a filtered regular Higgs bundle $E$, a filtered regular $\mathscr{D}_{X}$-module $V$, and a filtered local system $L$ correspond in the above correspondence, then there are isomorphisms

$$
\operatorname{res}_{s}(E) \cong \operatorname{res}_{s}(V) \cong \operatorname{res}_{s}(L)
$$

which respect the finer decomposition according to grading and eigenvalue, with a permutation of the indices as described in the table below; and such that on 
each piece of the decomposition, the nilpotent parts of the residual endomorphisms are equal. The permutations of the jumps in the filtrations and the eigenvalues of the endomorphism are given by this table:

\begin{tabular}{|c|c|c|c|}
\hline & $(E, \theta)$ & $(V, \nabla)$ & $L$ \\
\hline jump & $\alpha$ & $\alpha-2 b$ & $\beta=-2 b$ \\
\hline eigenvalue & $b+c i$ & $\alpha+2 c i$ & $\exp (-2 \pi i \alpha+4 \pi c)$ \\
\hline
\end{tabular}

A more combinatorial way to formalize this is the following. For each of the points $s$, form a residue diagram. This consists of a collection of partitions $P_{s}^{u, v}$ which together total $n$, labeled by pairs consisting of a real number $u$, the jump, and a complex number $v$, the eigenvalue. The partitions describe the type of the nilpotent part of the residual endomorphism, acting on its $v$ generalized eigenspace in the $u$ piece of the grading of the residue space at $s$. The theorem is that, under the correspondences, the residue diagrams remain the same but with labeling permuted as described in the table.

The residues also describe the behavior of the harmonic map of the corresponding tame harmonic bundle. This happens through the mechanism of weights of a nilpotent endomorphism. Given a nilpotent endomorphism $N$ of a vector space, put it in Jordan normal form and assign to the basis vectors integer weights so that $N$ lowers weights by 2 , and so that the weights of each Jordan block are arranged symmetrically about the origin. For example if $N$ is a nonzero nilpotent two-by-two matrix, the weights would be 1 and -1 . The Jordan normal form is not canonical, but the weight filtration is canonical: $W_{k}$ is the span of the vectors of weights less than or equal to $k$. This construction gives a weight filtration of the residues defined above; namely, on each subspace of the decomposition by grading and eigenvalue, take the weight filtration of the nilpotent part of the residual endomorphism.

The weight filtration of the residue $\operatorname{res}_{s}(L)$ of the filtered local system describes the behavior of the harmonic map at the puncture $s$, as follows. Choose a basis of $\operatorname{res}_{s}(L)$ compatible with the decomposition into $\operatorname{res}_{s}(L)_{\beta}$ (associated graded of the structural filtration), and compatible with the weight filtration. Then form a diagonal matrix $F(r)$ with entries $r^{\beta}|\log | r^{k / 2}$ for the basis elements in $\operatorname{res}_{s}(L)_{\beta}$ of weight $k$. Fix a ray emanating outward from $s$, lift it to the universal cover, and call it $\rho$. The distance $r$ to the puncture (in a euclidean metric on $\bar{X}$ ) gives the linear coordinate on the ray $\rho$. The matrix $F(r)$ gives a map from $\rho$ to $\mathrm{Gl}(n, \mathrm{C}) / \mathrm{U}(n)$. After a suitable automorphism of the symmetric space corresponding to the change of basis, the values of the harmonic map along the ray $\rho$ lie within a uniformly bounded distance from the values of this map $F(r)$, if the distance in the symmetric space is measured using an invariant metric.

Another way to say essentially the same thing is to say that the filtration $L_{\beta}$ and the weight filtration describe the norms of flat sections along the ray $\rho$, as 
measured by the harmonic metric accorded to the local system by the harmonic bundle. If $l$ is a flat section in $L_{\beta}$ but not in a smaller level of the filtration, and if the image of $l$ in the associated graded $\operatorname{res}(L)_{\beta}$ lies in $W_{k}$ but not in $W_{k-1}$, then the norm of $l$ measured in the harmonic metric is within a bounded multiple of $r^{\beta}|\log r|^{k / 2}$.

Similar statements hold for holomorphic sections of the filtered Higgs bundle and filtered $\mathscr{D}_{X}$-module. The description in the latter case is the same as the following description in the former. If $E$ is a filtered regular Higgs bundle coming from a harmonic bundle, then $E$ has a smoothly varying metric harmonic metric over $X$. The filtration $E_{\alpha, s}$ and the weight filtration of $\operatorname{res}_{s}(E)$ describe the norms of meromorphic sections of $E$ near $s$. If $e$ is a meromorphic section of $E$, then it lies in $E_{\alpha, s}$ for some $\alpha$; take the smallest $E_{\alpha, s}$ (the biggest $\alpha)$. Then look at the image of $e$ in the associated graded $\operatorname{res}_{s}(E)_{\alpha}$. We can assume that $\alpha$ is between 0 and 1 by multiplying by an appropriate power of $z$. If the image of $e$ lies in $W_{k}$ but not in $W_{k-1}$, then the norm of $e$ measured in the harmonic metric is within a bounded multiple of $r^{\alpha}|\log r|^{k / 2}$.

Finally let me describe the role played by some particular types of objects. Principal among the examples of harmonic bundles which come from geometry are Griffiths' variations of Hodge structure. A complex variation of Hodge structure is a local system with a smoothly varying decomposition $H=\bigoplus H^{p}$, with a locally constant hermitian form which is $(-1)^{p}$-definite on the orthogonal spaces $H^{p}$, such that the flat connection satisfies the transversality condition

$$
D: H^{p} \rightarrow A^{1,0}\left(H^{p}\right) \oplus A^{1,0}\left(H^{p-1}\right) \oplus A^{0,1}\left(H^{p}\right) \oplus A^{0,1}\left(H^{p+1}\right) .
$$

The associated positive definite metric obtained by changing the signs on $H^{p}$ is a harmonic metric for the local system. The harmonic map in the resulting harmonic bundle is a projection of Griffiths' classifying map, which maps $\widetilde{X}$ into a complex analytic symmetric space called the period domain, to the symmetric space $\mathrm{Gl}(n) / \mathrm{U}(n)$. In the filtered local system obtained by our correspondence, the filtrations at the punctures are trivial.

The filtered regular Higgs bundles which come from complex variations of Hodge structure are of the following special kind. A filtered regular system of Hodge bundles is a filtered regular Higgs bundle which has a decomposition $E=\oplus E^{p}$ of filtered vector bundles, such that $\theta$ has degree -1 in this grading:

$$
\theta: E^{p} \rightarrow E^{p-1} \otimes \Omega_{X}^{1}
$$

( $\theta$ satisfying as well the regularity conditions with respect to the filtrations). Under our correspondence, the harmonic bundles which come from irreducible complex variations of Hodge structure correspond to the stable filtered regular systems of Hodge bundles of degree zero. A useful point is that, when checking stability, only subsystems of Hodge bundles need be considered.

The system of Hodge bundles corresponding to a variation of Hodge structure may be described as follows. The transversality condition implies that the 
Hodge filtration $F^{p}=\bigoplus_{r \geq p} H^{r}$ varies holomorphically. Set $E^{p}=F^{p} / F^{p-1}$. The second fundamental form describing how the filtration varies provides the map $\theta: E^{p} \rightarrow E^{p-1} \otimes \Omega_{X}^{1}$. There are extensions $E_{\alpha, s}^{p}$ which are compatible with the Hodge decomposition, giving a structure of filtered regular system of Hodge bundles. The existence of these extensions and the fact that $\theta$ satisfies the regularity condition were first proved for the case of variations of Hodge structure many years ago by Griffiths and Schmid. Also proved by Schmid in this case was the statement that the nilpotent part of the monodromy transformation of the local system is the same as the (automatically nilpotent) residue of $\theta$.

There is an action of $\mathbf{C}^{*}$ on the set of stable filtered regular Higgs bundles of degree zero: $t \in \mathbf{C}^{*}$ sends $(E, \theta)$ to $(E, t \theta)$, keeping the same filtration. The fixed points of this action are the stable filtered regular systems of Hodge bundles of degree zero, in other words the Higgs bundles which come from (irreducible) variations of Hodge structure as above.

Via our correspondence, $\mathbf{C}^{*}$ acts on the space of stable filtered local systems, and the fixed points are the local systems which come from variations of Hodge structure. The filtrations and the norms of the eigenvalues are changed by the action; any fixed points must have trivial filtrations and eigenvalues of norm 1. Conversely, if the filtrations of a filtered local system are trivial, and the eigenvalues of the monodromy transformations have absolute value 1 , then the action of $\mathbf{C}^{*}$ preserves the isomorphism classes of the residues, and so the conjugacy classes of the monodromy transformations. If such an irreducible local system is strongly rigid for specified conjugacy classes of monodromy, in other words if the local system is uniquely determined by the conjugacy classes of its monodromy transformations around the punctures, then it must come from a complex variation of Hodge structure.

\section{HARMONIC BUNDLES}

In this section we will recall some basic notations about harmonic bundles. Throughout the paper, $X$ is a noncompact curve, and $\omega$ is a metric which extends smoothly to the compact completion $\bar{X}$.

Locally, a harmonic bundle is the same thing as a harmonic map from an open set in $X$ to the homogeneous space $\mathrm{Gl}(n) / \mathrm{U}(n)$. We will think of the harmonic bundle as a constant vector space together with a smoothly varying metric $K$, satisfying some equations. A point in $\mathrm{Gl}(n) / \mathrm{U}(n)$ represented by a matrix $h$ corresponds to the metric $(u, v)_{K}=\left({ }^{t} h h u, v\right)$ on $\mathbf{C}^{n}$, the latter being the standard metric. Globally, a harmonic bundle is a flat bundle with a smoothly varying metric, which locally satisfies the equations of a harmonic map. Alternatively one may think of a representation $\pi_{1}(X) \rightarrow \mathrm{Gl}(n, \mathrm{C})$ (the monodromy representation of the flat bundle), and an equivariant harmonic map from the universal cover of $X$ to the space $\mathrm{Gl}(n) / \mathrm{U}(n)$.

There is another type of object with metric which turns out to be equivalent. A Higgs bundle is a holomorphic bundle $(E, \bar{\partial})$ with a holomorphic 
endomorphism-valued one-form $\theta: E \rightarrow E \otimes \Omega_{X}^{1}$. We can consider smoothly varying metrics on a Higgs bundle, and if certain additional equations are satisfied, such objects are the same as harmonic bundles.

The equations which are required of the harmonic metrics in either of the cases, and the resulting equivalence, were discussed in detail in [20]. They are local matters, so the discussion carries over to here. We will simply review the results and the notation.

If $(V, D)$ is a bundle with flat connection, we denote the decomposition of the connection into operators of types $(1,0)$ and $(0,1)$ by

$$
D=d^{\prime}+d^{\prime \prime} \text {. }
$$

Given a metric on a flat bundle $(V, D)$, one obtains (by linear algebra) some other operators $\bar{\partial}, \partial, \theta$, and $\bar{\theta}$, of types $(0,1),(1,0),(1,0)$, and $(0,1)$ respectively. These automatically satisfy the following conditions. $\partial+\bar{\partial}$ respects the metric, in other words $(\partial u, v)+(u, \bar{\partial} v)=\partial(u, v)$; we have $(u, \theta v)$ $=(\bar{\theta} u, v)$. We have $d^{\prime \prime}=\bar{\partial}+\bar{\theta}$ and $d^{\prime}=\partial+\theta$. Setting $\delta^{\prime}=\partial-\theta$ and $\delta^{\prime \prime}=\bar{\partial}-\bar{\theta}$, the connections $\delta^{\prime}+d^{\prime \prime}$ and $d^{\prime}+\delta^{\prime \prime}$ respect the metric $K$. Form the operators $D^{\prime \prime}=\bar{\partial}+\theta$ and $D^{\prime}=\partial+\bar{\theta}$. The pseudo-curvature of the metric is the endomorphism-valued two-form

$$
G_{K}=\left(D^{\prime \prime}\right)^{2}=\bar{\partial}(\theta) \text {. }
$$

Note that here, since $X$ has complex dimension one, we have $\bar{\partial}^{2}=0$ and $\theta^{2}=0$. The condition for the metric to be harmonic is $G_{K}=0$. If this condition holds, then the operator $\bar{\partial}$ gives a holomorphic structure, and $\theta$ is holomorphic, so we get a metrized Higgs bundle.

The construction works similarly in the reverse. Suppose $(E, \theta)$ is a Higgs bundle. Let $\bar{\partial}$ denote the holomorphic structure. Suppose $E$ has a metric. Then we get operators $\partial, \bar{\theta}, d^{\prime}, d^{\prime \prime}, D$, and so forth. These are defined by the condition that $\partial+\bar{\partial}$ preserves the metric, and that $(u, \theta v)=(\bar{\theta} u, v)$. Then $d^{\prime \prime}=\bar{\partial}+\bar{\theta}, d^{\prime}=\partial+\theta$, and $D=d^{\prime}+d^{\prime \prime}$. The curvature of the metric is the endomorphism-valued two-form

$$
F_{K}=D^{2}=\partial \bar{\partial}+\bar{\partial} \partial+\theta \bar{\theta}+\bar{\theta} \theta .
$$

It is the curvature of the connection $D$. Note that the expression is again simplified since $X$ is a curve. The condition for the metric to be harmonic is that $F_{K}=0$. If this holds, then $D$ is flat connection.

These constructions are inverses: if $(V, D)$ is a flat bundle and $K$ is a harmonic metric, then the same metric $K$ is a harmonic metric for the resulting Higgs bundle; and the flat connection induced by this metric on the Higgs bundle is equal to the original. Similarly if $(E, \theta)$ is a Higgs bundle and $K$ is a harmonic metric, then the same metric is a harmonic metric on the flat bundle, and the resulting Higgs structure is the same as before.

Tameness. We will only consider certain types of harmonic bundles, those satisfying a growth property we call tameness. It is actually a property of the Higgs 
bundle underlying a harmonic bundle. The endomorphism-valued one-form $\theta$ has eigenvalues, which are multivalued holomorphic one-forms on $X$ (with finitely many branches). The definition is that $(E, \theta)$ is tame if the eigenvalues of $\theta$ have poles of order at most one, at the puncture points. (Note that the poles can have fractional order, depending on the multiplicity of values of $\theta$.) We will see at the end of $\S 2$ that this definition of tameness agrees with the definition involving polynomial growth of the metric, which was given in the Synopsis.

The restriction to tame harmonic bundles seems to be necessary for getting good estimates like those of $\$ 2-i$ is an interesting problem for further study to remove the restriction. Fortunately, our restricted class of tame harmonic bundles corresponds nicely with topological objects, as we will see by the end of the paper.

Regular singularities. A harmonic bundle has associated an analytic object, a locally free $\mathscr{O}_{X^{-}}$-module $V$ with integrable connection. Such objects are $\mathscr{D}_{X^{-}}$ modules of the simplest type, and we will abuse notation and use the terminology " $\mathscr{D}_{X}$-module" to mean just these particular ones. The notion of $\mathscr{D}_{X}$-module means that there is an action of the sheaf of differential operators. This action is given by a connection, which specifies the action of the vector fields. The other differential operators are sums of compositions of derivations given by vector fields. A connection is a map $\nabla: V \rightarrow V \otimes \Omega_{X}^{1}$, and the integrability condition $\nabla^{2}=0$ (automatic since $X$ is a curve) insures that it gives a well-defined action of differential operators of higher orders.

If $\left(E, D, D^{\prime \prime}, K\right)$ is a harmonic bundle on $X$, then the flat analytic connection $D$ gives an analytic $\mathscr{D}_{X}$-module $V$. The holomorphic structure of the holomorphic bundle underlying this $\mathscr{D}_{X}$-module is given by the operator $d^{\prime}$, the part of $D$ of type $(0,1)$. The holomorphic bundle $V=\left(E, d^{\prime \prime}\right)$ has a holomorphic connection $\nabla=d^{\prime}$, the part of $D$ of type $(1,0)$.

An analytic $\mathscr{D}_{X}$-module is the same thing as a local system of $\mathbf{C}$ vector spaces on $X$, in other words a representation of the fundamental group. This correspondence is given as follows. If $V$ is locally free over $\mathscr{O}_{X}^{\text {an }}$ with flat connection $\nabla$, then $L=V^{\nabla}$, the sheaf of sections killed by $\nabla$, is a locally constant sheaf in the usual topology of $X^{\text {an }}$. It is the local system of solutions of the ordinary differential equation $\nabla(v)=0$. The representation of $\pi_{1}(X)$ is obtained by continuing a solution at $x$ around a loop and back to $x$. If $(V, \nabla)$ is the $\mathscr{D}_{X}$-module coming from a harmonic bundle $\left(E, D, D^{\prime \prime}, K\right)$, then the associated local system is the same as the local system of flat sections of $E$ with respect to the flat differential geometric connection $D$.

The notion of $\mathscr{D}_{X}$-module makes sense in the algebraic category, too. There is a notion of regular singularities for algebraic $\mathscr{D}_{X}$-modules on $X$. A reference is [4], although most books on ordinary differential equations discuss this concept. It depends strongly on the algebraic structure of $V$ at the punctures. If $V$ starts as an analytic locally free sheaf on $X$, then to give an algebraic 
structure at a puncture essentially means to specify which holomorphic sections are meromorphic at the puncture. The connection $\nabla$ has regular singularities if the flat sections along rays out to the puncture grow at most polynomially when expressed in terms of a basis of meromorphic sections of $V$. An equivalent formulation is that $\nabla$ has regular singularities if there exists an extension $\bar{V}$ of $V$ to a locally free sheaf on the smooth completion $\bar{X}$, compatible with the meromorphic structure of $V$, and such that when the connection is written by a connection matrix $\nabla=d+A$ with respect to a framing of the locally free sheaf $\bar{V}$, the matrix $A$ consists of one-forms with poles of order $\leq 1$. It should be noted that $\bar{V}$ is not unique, and that not every extension will do. The $\mathscr{D}_{X}$-modules with which we will deal in this paper will always have regular singularities.

It seems like a good idea to discuss the relationship between the module with connection and the local system near a puncture, in the case of regular singularities. Work over a punctured disk, with coordinate $z$. The flat sections of $V$, those killed by $\nabla$, are multivalued holomorphic sections of $V$ over the punctured disk. The matrix expressing what happens to a basis of sections upon continuing once around is called the monodromy matrix $\mu$. More invariantly, suppose $\rho$ is a ray going out from the puncture (e.g. the positive real axis). Then we can speak of the space of flat sections along the ray, a vector space $L(\rho)$. It has the same dimension as the rank of the bundle $V$. The process of continuing the solutions of the ordinary differential equation gives the monodromy transformation $\mu: L(\rho) \rightarrow L(\rho)$.

There is a somewhat canonical way to create single valued holomorphic sections of $V$, by untwisting the monodromy transformation. It depends on the coordinate $z$, as well as on a choice of logarithm of the monodromy transformation, more precisely a matrix $M$ such that $e^{2 \pi i M}=\mu$. If $l \in L(\rho)$, continue it to a multivalued section $l(z)$. Set

$$
h(z)=e^{-M \log z} l(z) .
$$

It is single valued, because upon continuing around, $l$ goes to $\mu l$, and $e^{-M \log z}$ goes to $e^{-M \log z} e^{-2 \pi i M}$, so $h$ stays the same.

As $l$ runs through a basis of $L(\rho)$, the $h$ provide a frame for a holomorphic extension $\bar{E}$ of the bundle. Let us calculate the connection matrix:

$$
\begin{gathered}
\begin{aligned}
& \nabla(h)=d\left(e^{-M \log z}\right) l+e^{-M \log z} \nabla(l), \\
& \text { but } \nabla(l)=0 \text { and } d\left(e^{-M \log z}\right)=-M d z / z e^{-M \log z}, \text { so } \\
& \nabla(h)=-M \frac{d z}{z} h .
\end{aligned}
\end{gathered}
$$

Thus the matrix for $\nabla$ in this frame is $-M d z / z$. Notice that this shows that any analytic $\mathscr{D}_{X}$-module on $X$ has an algebraic structure (given by the extension $\bar{V}$ defined above) in which $\nabla$ has regular singularities. Conversely, if $V$ is algebraic and $\nabla$ has regular singularities, then the sections $h$ defined above will be meromorphic [4]. 
We will see that if $\left(E, D, D^{\prime \prime}, K\right)$ is a tame harmonic bundle, then the holomorphic bundle $V$ has an algebraic structure given by the metric $K$ (due to the estimates on the curvature of this metric given in the next section). With respect to this algebraic structure, $\nabla$ will have regular singularities. A sort of converse will be given by Proposition 2.1. Although the existence of an algebraic structure given by $K$ is not a priori clear without the hypothesis of tameness, we can still use the metric to measure the growth of sections. Proposition 2.1 will say that if the flat sections of $E$ have polynomial growth as measured by $K$, then the harmonic bundle is tame.

\section{Main estimate}

In this section we will give the main estimate for the growth of the curvature of a metric on a tame harmonic bundle. We start with a definition of tameness based on the eigenvalues of $\theta$, and at the end we will prove that this definition is equivalent to the definition in terms of polynomial growth of the harmonic metric.

The situation is as follows. Suppose $E$ is a harmonic bundle over the punctured unit disk $B^{*}$ in the complex plane. Keep the notations of $\S 1$, and use the euclidean metric $d z d \bar{z}$ on $B^{*}$. Here $z$ is the standard coordinate. As a matter of notation, $r$ always denotes the radius $|z|$ from the puncture. We will be cavalier in neglecting what happens at the outer boundary $r=1$.

Make the assumption that $E$ is tame, in other words that the eigenvalues of $\theta$ are bounded $\left|\lambda_{i}\right| \leq C / r$. The eigenvalues of $\theta$ are (multivalued) holomorphic functions, and we may divide up the eigenvalues into groups $\lambda_{1}^{i}, \ldots, \lambda_{e_{i}}^{i}$ and so on, with distinct numbers $a_{i}$ so that

$$
\left|\lambda_{j}^{i}-a_{i} \frac{d z}{z}\right| \leq \frac{C}{r^{1-\varepsilon}} .
$$

Note that $\varepsilon$ can be taken as $1 / n$ where $n$ is the rank of $E$. While the eigenvalues themselves are multivalued, the groups of eigenvalues according to their residues are well defined. $E$ decomposes holomorphically $E=\bigoplus E_{i}$ such that $E_{i}$ is the sum of the generalized eigenspaces for eigenvalues $\lambda_{j}^{i}$. Define a new endomorphism-valued one-form $\phi$, semisimple with eigenvalues $a_{i} d z / z$, and eigenspaces $E_{i}$. The subspaces $E_{i}$ are preserved by $\phi$ and $\theta$.

Choose an ordering of the $E_{i}$ and make a filtration; then fill in this filtration to a complete flag, a filtration where the quotients have rank 1 , of subbundles of $E$, preserved by $\theta$. One may not be able to do this holomorphically on the disc, for the eigenspaces of $\theta$ may be multivalued. They have finite branching, so we can choose the filtration after passing to a finite ramified cover $z=u^{N}$. Alternatively, we may do it on any angular sector.

Choose a basis $\left\{e_{k}\right\}$ of $E$, orthonormal with respect to the harmonic metric, and compatible with the flag, which is to say that subbundles in the flag are obtained as the spans of vectors in the basis in some order. Write everything in 
terms of this basis. We have

$$
\theta=\sigma+\tau, \quad \phi=\alpha+q,
$$

where $\sigma$ and $\alpha$ are diagonal matrices of one-forms, and $\tau$ and $q$ are upper triangular matrices of one-forms. The entries of the diagonal $\alpha$ are $a_{i} d z / z$. Furthermore, the entries of $\sigma$ are the eigenvalues $\lambda_{j}^{i}$, so we can write

$$
\sigma=\alpha+\beta
$$

with estimates

$$
|\alpha| \leq C r^{-1}, \quad|\beta| \leq r^{-1+\varepsilon} .
$$

Since the $E_{i}$ are single valued, $\alpha$ and $\phi$ are single valued. Thus $q$ and $\beta+\tau$ are single valued, even though $\beta$ and $\tau$ may individually be multivalued.

Decompose things further with respect to the block form provided by the subbundles $E_{i}$. Let

$$
\begin{aligned}
M^{+} & =\bigoplus_{i>j} \operatorname{Hom}\left(E_{i}, E_{j}\right) \subset \operatorname{End}(E), \\
M^{0} & =\bigoplus_{i} \operatorname{End}\left(E_{i}\right) \subset \operatorname{End}(E), \\
M^{-} & =\bigoplus_{i<j} \operatorname{Hom}\left(E_{i}, E_{j}\right) \subset \operatorname{End}(E) .
\end{aligned}
$$

In other words, $M^{+}$consists of the block upper triangular matrices, $M^{-}$the block lower triangular matrices, and $M^{0}$ the block diagonal matrices.

Note that $q$ is already in $M^{+}$. To see this, let $V_{i}$ be the space spanned by the basis elements which correspond to the subquotient $E_{i}$ of the filtration. Then $\bigoplus_{i<j} E_{i}=\bigoplus_{i<j} V_{i}$ (call this $F_{j}$ ). Now $V_{i}$ are the eigenspaces of $\alpha$, whereas $E_{i}$ were the eigenspaces of $\phi$. Both $\alpha$ and $\phi$ preserve the filtration $F_{j}$, and act by $a_{i} d z / z$ on $\operatorname{Gr}_{i}(F)$. Therefore $q=\phi-\alpha$ sends $F_{i}$ into $F_{i-1}$, in other words it is block upper triangular.

Write $\tau=\tau^{0}+\tau^{+}$with $\tau^{+}$in $M^{+}$and $\tau^{0}$ upper triangular in $M^{0}$. The spaces $M^{0}$ and $M^{+}$are single valued, so $\tau^{+}$and $\beta+\tau^{0}$ are single valued.

Theorem 1. We have the following bounds for the above objects associated with the tame harmonic bundle $E$ (constants are implied):

$$
\begin{gathered}
\left|\tau^{0}\right| \leq r^{-1}|\log r|^{-1}, \\
\left|\tau^{+}\right| \leq r^{-1+\varepsilon}, \quad|q| \leq r^{-1+\varepsilon} .
\end{gathered}
$$

Let $\mu=\partial+\bar{\partial}$ denote the metric connection on the bundle with respect to the operator $\bar{\partial}$, let $\nu=d^{\prime}+d^{\prime \prime}-2 \theta$ denote the other metric connection, corresponding to the bundle underlying the $\mathscr{D}_{X}$-module, and let $R_{\mu}$ and $R_{\nu}$ denote the curvatures of these connections. Then

$$
|R| \leq \frac{C}{r^{2}|\log r|^{2}}
$$


for both curvatures $R=R_{\mu}$ and $R=R_{\nu}$. Also, the norm of $\theta$ is bounded by $C / r$.

Proof. The first estimates are the main point. The last statement then follows: note that the curvatures $R$ are both multiples of $\theta \bar{\theta}+\bar{\theta} \theta$. The matrix for $\bar{\theta}$ is plus or minus the transpose complex conjugate of the matrix for $\theta$. Also, $\alpha$ commutes with $\bar{\alpha}$, and with $\tau^{0}$ and $\bar{\tau}^{0}$. Therefore the inequalities $|R| \leq$ $r^{-2}|\log r|^{-2}$ follow from the first inequalities. The last line of the theorem is obvious.

The next several pages will be devoted to the proof of the initial inequalities. If one wants to make the first statement single valued, it may be rephrased as $\left|\beta+\tau^{0}\right| \leq r^{-1}|\log r|^{-1}$. It suffices to prove the theorem after pulling back to the ramified cover $z=u^{N}$. This is because (up to constants) one-forms $d z /|z|$ transform to $d u /|u|$, two-forms $d z d \bar{z} /|z|^{2}$ transform to $d u d \bar{u} /|u|^{2}$, and the logarithm terms remain the same. Henceforth assume that we have made this reduction and called $u \quad z$ again, so that everything becomes a single valued function of $z$. First we will obtain the estimate for $\left|\tau^{0}\right|$ using Ahlfors' method, generalizing the estimates involved in the distance decreasing property of variations of Hodge structure.

The following are some preliminary curvature calculations. The curvature of the unitary bundle $($ End $V) \otimes \Omega_{X}^{1}$ is

$$
\nabla^{2}=\operatorname{ad}\left(R_{\mu}\right) \otimes 1,
$$

where $R_{\mu}=\mu^{2}$ is the curvature of the metric connection $\mu=\partial+\bar{\partial}$, noting that the curvature of $\Omega_{X}^{1}$ is zero since we use the euclidean metric. The statement that curvature decreases in subbundles, applied to the subline bundle of $($ End $V) \otimes \Omega_{X}^{1}$ given by $\theta$, can be written

$$
\Delta \log |\theta|^{2} \leq \frac{\left(2 \sqrt{-1} \Lambda \nabla^{2}(\theta), \theta\right)}{|\theta|^{2}}
$$

This holds in a distributional sense everywhere.

Now note that $R_{\mu}+\theta \bar{\theta}+\bar{\theta} \theta=0$. Therefore

$$
\Delta \log |\theta|^{2} \leq-\frac{(2 \sqrt{-1} \mathrm{ad} \Lambda(\theta \bar{\theta}+\bar{\theta} \theta)(\theta), \theta)}{|\theta|^{2}} .
$$

Now, at any point, if we write $\theta=\theta_{0} d z$ and $\bar{\theta}=\bar{\theta}_{0} d \bar{z}$ in a normal coordinate $z$, then $2 \sqrt{-1} \Lambda(\theta \bar{\theta}+\bar{\theta} \theta)=\left[\theta_{0}, \bar{\theta}_{0}\right]$ and

$$
\begin{aligned}
\frac{(2 \sqrt{-1} \operatorname{ad} \Lambda(\theta \bar{\theta}+\bar{\theta} \theta)(\theta), \theta)}{|\theta|^{2}} & =\frac{\left(\left[\left[\theta_{0}, \bar{\theta}_{0}\right], \theta_{0}\right], \theta_{0}\right)}{\left|\theta_{0}\right|^{2}} \\
& =\frac{\left|\left[\theta_{0}, \bar{\theta}_{0}\right]\right|^{2}}{\left|\theta_{0}\right|^{2}} .
\end{aligned}
$$


For brevity, let us abuse notation and write $[\theta, \bar{\theta}]$ instead of $\left[\theta_{0}, \bar{\theta}_{0}\right]$. Our curvature calculation becomes

$$
\Delta \log |\theta|^{2} \leq-\frac{|[\theta, \bar{\theta}]|^{2}}{|\theta|^{2}} .
$$

By our assumptions on the eigenvalues of $\theta$, we may write $\sum\left|\lambda_{j}^{i}\right|^{2}=a / r^{2}+b$, where $|b| \leq C / r^{2-\varepsilon}$, so we have $|\sigma|^{2}=a / r^{2}+b$. Note that $|\theta|^{2}=|\sigma|^{2}+|\tau|^{2}$.

We next claim that $|[\tau, \bar{\tau}]| \geq c|\tau|^{2}$. The argument is the same as in the case of Hodge structures [11]; we will sketch it. Note that the matrix for $\bar{\tau}$ is the transpose complex conjugate of the matrix for $\tau$, in particular it is lower triangular. The upper left entry of $[\tau, \bar{\tau}]$ is the square norm of the first row of $\tau$. If this is as big as the square norm of $\tau$, we are done. If it is much smaller than the square norm of $\tau$, then the contribution from the first row to the next entry on the diagonal of $[\tau, \bar{\tau}]$ is small, so this second diagonal entry is approximately the square norm of the second row of $\tau$. Continue in this fashion until reaching a row whose norm is comparable to the norm of $\tau$. This proves the claim.

From this claim we get the estimate

$$
\Delta \log \left(|\sigma|^{2}+|\tau|^{2}\right) \leq-c \frac{|\tau|^{4}}{|\sigma|^{2}+|\tau|^{2}} .
$$

We will make use of this estimate and follow Ahlfors' method [1] in order to estimate the quantity $|\tau|^{2}$. The basic idea is that if there is an inequality involving the laplacian, one should consider auxiliary functions satisfying the opposite inequality. The maximum principle allows one to compare the original function to the auxiliary function. The auxiliary function should blow up faster (or earlier) than the original function, as $r \rightarrow 0$. In the process of doing this, we will sometimes want to be more precise about constants, in which case they will be numbered.

Step 1. We will prove that $|\tau| \leq C / r$. Set $h=|\sigma|^{2}+|\tau|^{2}$. Since $|\sigma|^{2} \leq C / r$, there are constants $C_{0}$ and $c_{1}$ such that if $h \geq C_{0} / r^{2}$, then $|\tau|^{4} \geq c h^{2}$, so

$$
\Delta \log h \leq-c_{1} h .
$$

Choose $C_{2}$ so that $C_{2} \geq C_{0}$ and $2 / C_{2} \leq c_{1}$. Let $m_{\varepsilon}$ denote the auxiliary function $m_{\varepsilon}=C_{2} /(r-\varepsilon)^{2}$. Recall that on rotationally symmetric functions,

$$
\Delta=-\frac{\partial^{2}}{\partial r^{2}}-\frac{1}{r} \frac{\partial}{\partial r} \text {. }
$$

Therefore

$$
\begin{aligned}
\Delta \log m_{\varepsilon} & =-\left(\frac{\partial}{\partial r}+\frac{1}{r}\right)\left(\frac{-2}{r-\varepsilon}\right) \\
& =\frac{-2}{(r-\varepsilon)^{2}}+\frac{2}{r(r-\varepsilon)} \geq-\frac{2}{C_{2}} m_{\varepsilon} .
\end{aligned}
$$


Thus we get the estimate

$$
\Delta \log m_{\varepsilon} \geq-c_{1} m_{\varepsilon} .
$$

At any interior point with $h>m_{\varepsilon}$, we have in particular $h \geq C_{0} / r^{2}$, so

$$
\Delta \log \left(h / m_{\varepsilon}\right) \leq-c_{1}\left(h-m_{\varepsilon}\right)<0 \text {. }
$$

Therefore $h / m_{\varepsilon}$ cannot achieve a maximum value greater than 1 in the interior. On the other hand, $m_{\varepsilon}$ blows up at the inner boundary $r=\varepsilon$, so $h / m_{\varepsilon}$ does not achieve its maximum there and $h$ is bounded at the outer boundary $r=1$. In any case we conclude that

$$
h \leq C_{3} m_{\varepsilon} \text {. }
$$

The constant $C_{3}$ depends on the outer boundary values of $h$. Letting $\varepsilon \rightarrow 0$, we get $h \leq C / r^{2}$, which proves Step 1 .

Step 2. We prove that $|\tau| \leq C / r|\log r|$. Make the assumption that $a \neq 0$. If $a=0$ then $|\sigma| \leq C / r^{1-\varepsilon}$, and we may proceed in a similar but easier fashionthis is just the standard case of Ahlfors' lemma (cf. [19]). With $a \neq 0$ we may look at

$$
k=\log \frac{r^{2}}{a}\left(|\sigma|^{2}+|\tau|^{2}\right) .
$$

Recall that $|\sigma|^{2}=a / r^{2}+b$ with $|b| \leq C / r^{2-\varepsilon}$. Therefore

$$
k=\log \left(1+r^{2} b / a+r^{2}|\tau|^{2} / a\right),
$$

so

$$
c\left(r^{2} b+r^{2}|\tau|^{2}\right) \leq k \leq C\left(r^{2} b+r^{2}|\tau|^{2}\right),
$$

and in particular $0 \leq k \leq C$. It will suffice to prove that $k \leq C /|\log r|^{2}$.

Since $|b| \leq C / r^{2-\varepsilon}$, there is a constant $C_{0}$ such that if $k \geq C_{0} /|\log r|^{2}$, then $|\tau|^{2}$ is bigger than $b$, so $|\tau|^{4} \geq c k^{2}$. Thus, at any point where $k \geq C_{0} /|\log r|^{2}$,

$$
\Delta k \leq-c_{1} \frac{k^{2}}{r^{2}} .
$$

We may make $c_{1}$ smaller so that $6 / c_{1} \geq C_{0}$. Choose an auxiliary function

$$
p_{\varepsilon}=\frac{C_{2}}{|\log r|^{2}}+\varepsilon|\log r| \text {. }
$$

We have

$$
\begin{aligned}
\Delta p_{\varepsilon} & =-\left(\frac{\partial}{\partial r}+\frac{1}{r}\right)\left(\frac{2 C_{2}}{C r|\log r|^{3}}\right) \\
& =\frac{-6 C_{2}}{r^{2}|\log r|^{4}} \geq-\frac{6 p_{\varepsilon}^{2}}{C_{2} r^{2}} .
\end{aligned}
$$

Setting $C_{2}=6 / c_{1}$ we get

$$
\Delta p_{\varepsilon} \geq-c_{1} \frac{p_{\varepsilon}^{2}}{r^{2}}
$$


Since $C_{2} \geq C_{0}$, at any point where $k-p_{\varepsilon}>0$ we have $k \geq C_{0} /|\log r|^{2}$. So the above estimate on $\Delta k$ gives

$$
\Delta\left(k-p_{\varepsilon}\right) \leq-c_{1} \frac{k^{2}-p_{\varepsilon}^{2}}{r^{2}}<0 .
$$

Therefore $k-p_{\varepsilon}$ cannot achieve a maximum value greater than zero in the interior. On the other hand, $k$ is bounded where $p_{\varepsilon}$ blows up at $r=0$ and $r=1$, so $k \leq p_{\varepsilon}$. Letting $\varepsilon$ tend to zero, we get $k \leq C_{2} /|\log r|^{2}$. This proves Step 2, which completes the first of the estimates in the theorem.

Now we will turn to $\tau^{+}$and $q$. The idea is to use the same type of argument as above, but using curvature calculations involving $\phi$ instead of $\theta$.

First, use the equation $[\phi, \theta]=0$ to find the relationship between $\phi$ and $\theta$. Expressing them in terms of $\alpha, \beta, \tau$, and $q$, the equation becomes

$$
\operatorname{ad}(\alpha)(\tau)=(\operatorname{ad}(\alpha)+\operatorname{ad}(\beta+\tau))(q) \text {. }
$$

Note that $\operatorname{ad}(\alpha)(\tau)=\operatorname{ad}(\alpha)\left(\tau^{+}\right)$. Furthermore, the term $\operatorname{ad}(\beta+\tau)(q)$ must lie in the space $M^{+}$, so we may replace $\operatorname{ad}(\beta+\tau)$ by its composition with the projection to $M^{+}$. Thus we have

$$
\operatorname{ad}(\alpha)\left(\tau^{+}\right)=\left(\operatorname{ad}(\alpha)+\operatorname{ad}(\beta+\tau)^{+}\right)(q) \text {. }
$$

But $\operatorname{ad}(\alpha): M^{+} \rightarrow M^{+}$is invertible, and its inverse satisfies $\left|\operatorname{ad}(\alpha)^{-1}\right| \leq C r$. On the other hand, by our above estimates, $\operatorname{ad}(\beta+\tau)^{+}$has norm bounded by $C / r|\log r|$. Therefore

$$
\tau^{+}=(1+f) q,
$$

where $f$ is an operator with norm bounded by $C /|\log r|$. In particular, note that $|q| \leq C / r|\log r|$.

Now we will apply the same curvature calculations as above, but to the holomorphic endomorphism $\phi$ instead of $\theta$. We get the estimate

$$
\Delta \log |\phi|^{2} \leq-\frac{(2 \sqrt{-1} \operatorname{ad} \Lambda(\theta \bar{\theta}+\bar{\theta} \theta)(\phi), \phi)}{|\phi|^{2}} .
$$

The term on the right-hand side can be written as

$$
-\frac{([[\theta, \bar{\theta}], \phi], \phi)}{|\phi|^{2}}
$$

and, since $[\theta, \phi]=0$, this becomes (by the Jacobi identity and the infinitesimal invariance of the inner product)

$$
-\frac{([\phi, \bar{\theta}],[\phi, \bar{\theta}])}{|\phi|^{2}}
$$

so our estimate becomes

$$
\Delta \log |\phi|^{2} \leq-c \frac{|[\phi, \bar{\theta}]|^{2}}{|\phi|^{2}}
$$


Note that the denominator $|\phi|^{2}$ is a bounded (above and away from zero) multiple of $1 / r^{2}$, because $\alpha \neq 0$ (otherwise $M^{+}$would be zero and there would be nothing to prove) and $|q| \leq C / r|\log r|$. Now,

$$
[\phi, \bar{\theta}]=[\alpha, \bar{\theta}]+[q, \bar{\alpha}]+[q, \overline{\beta+\tau}] .
$$

The first term is lower triangular. In the upper triangular part we have $-\operatorname{ad}(\bar{\alpha})(q)$ $-\operatorname{ad}(\overline{\beta+\tau})(q)$. But $\operatorname{ad}(\bar{\alpha})$ has an inverse on $M^{+}$, with norm $\leq C r$, and the norm of the composition of $\operatorname{ad}(\overline{\beta+\tau})$ with projection to $M^{+}$is bounded by $C / r|\log r|$. Therefore,

$$
|\operatorname{ad}(\bar{\alpha})(q)+\operatorname{ad}(\overline{\beta+\tau})(q)| \geq c|q| / r .
$$

Thus, $|[\phi, \bar{\theta}]|^{2} \geq|q|^{2} / r^{2}$ and our estimate becomes

$$
\Delta \log |\phi|^{2} \leq-c|q|^{2} \text {. }
$$

On the other hand, $|\phi|^{2}=|\alpha|^{2}+|q|^{2}=\frac{a}{r^{2}}\left(1+r^{2}|q|^{2} / a\right)$. Our estimate becomes

$$
\Delta \log \left(1+r^{2}|q|^{2} / a\right) \leq-c|q|^{2} .
$$

Finally, note that $r^{2}|q|^{2}$ is bounded, and set $k=\log \left(1+r^{2}|q|^{2} / a\right)$. Then $k$ is comparable to $r^{2}|q|^{2}$, and we get the estimate

$$
\Delta k \leq-c k / r^{2}
$$

Fix a small number $u$ (which will depend on $c$ as we shall see in a minute). Consider the auxiliary function $p_{\varepsilon}=r^{u}+\varepsilon|\log r|$. Then

$$
\Delta p_{\varepsilon}=-\left(\frac{\partial}{\partial r}+\frac{1}{r}\right)\left(u r^{u-1}\right)=-(u(u-1)+u) r^{u-2}=-u^{2} r^{u-2} .
$$

Thus if $u^{2}<c$ we have

$$
\Delta p_{\varepsilon} \geq-c p_{\varepsilon} / r^{2}
$$

The auxiliary function blows up at the origin, but $k$ is bounded. In the usual way, we get the estimate $k \leq C r^{u}$, in other words, $|q|^{2} \leq C / r^{2-u}$. From above, this gives the same estimate for $\left|\tau^{+}\right|^{2}$. This completes the proof of the theorem, which is our main estimate.

Remark. The flat connection $d^{\prime}+d^{\prime \prime}$ differs from a unitary connection $\delta^{\prime}+d^{\prime \prime}$ by a matrix of norm less than $C / r$. Thus if $v$ is a flat section, we have

$$
\partial|v|^{2} / \partial r \leq C|v|^{2} / r,
$$

so $|v|^{2} \leq r^{C}$. The same holds for sections of the dual of the determinant bundle. Therefore the norms of the flat sections grow, and the determinant decreases, at most polynomially in the radius $r$. Conversely, if this condition holds then it implies that the harmonic bundle is tame, giving the following alternate characterization of tameness. 
Proposition 2.1. Suppose $V$ is a $\mathscr{D}_{X}$-module with a harmonic metric, and suppose that the norms of the flat sections grow (and the determinant decreases) at most polynomially. Then the eigenvalues of $\theta$ are bounded by $C / r$, so the harmonic bundle is tame and the above estimates apply.

Proof. We will think of a harmonic metric as an equivariant harmonic map to the space $G / U=\mathrm{Gl}(n) / \mathrm{U}(n)$ of metrics on a fixed vector space. Consider a circle $S_{\varepsilon}$ of radius $\varepsilon$ around the origin. If we fix boundary values equal to our original metric $K$ on $S_{\varepsilon}$ and $S_{1}$, then the metric $K$ is the metric which minimizes the energy $\int_{D(\varepsilon, 1)}|\theta|^{2}$ over the annulus $D(\varepsilon, 1)$ between the two circles. In particular, if we can find a different metric $H$ with the same boundary values, then we get an estimate

$$
\int_{D(\varepsilon, 1)}\left|\theta_{K}\right|^{2} \leq \int_{D(\varepsilon, 1)}\left|\theta_{H}\right|^{2}
$$

We will choose $H$ as follows. For each ray from the inner circle to the outer circle, we will define a map of the interval $[\varepsilon, 1]$ to the space $G / U$ of metrics (on the fixed vector space of flat sections along the ray). This will depend on an auxiliary number $\sigma$ which we may vary at the end. The map will send $[\varepsilon, \varepsilon+\sigma]$ to the geodesic whose endpoints are the inner boundary value and the outer boundary value. The map will be constant on the interval $[\varepsilon+\sigma, 1]$. Let us calculate the contribution to the energy from a small sector near one ray, including the rays whose angles are increased up to a small amount $d \phi$. Let $H_{0}$ and $H_{1}$ denote the two boundary values for the initial ray. Let $v_{0}$ denote the tangent vector at $H_{0}$ which is the velocity vector of the inner boundary values at $H_{0}$. Let $v_{1}$ denote the tangent vector at $H_{1}$. Then the corners of the sector are approximately $H_{0}, H_{0}+\varepsilon v_{0} d \phi, H_{1}+v_{1} d \phi$, and $H_{1}$. Note that $v_{1}$ is bounded. The contribution to the energy from $[\varepsilon+\sigma, 1]$ is

$$
\int_{\varepsilon+\sigma}^{1}\left|v_{1} / r\right|^{2} d \phi r d r \leq C|\log (\varepsilon+\sigma)| d \phi .
$$

The contribution to the energy from the interval $[\varepsilon, \varepsilon+\sigma]$ has two parts, coming from the radial and angular components of $\theta$. Let $L$ denote the length of the geodesic. Thus the speed is $L / \sigma$. The contribution from the radial component is

$$
\int_{\varepsilon}^{\varepsilon+\sigma}(L / \sigma)^{2} d \phi r d r \leq(\varepsilon+\sigma)(L)^{2} d \phi / \sigma .
$$

The tangential component of $|\theta|^{2}$ is bounded by $\sup \left(\left|v_{0}\right|^{2},\left|v_{1}\right|^{2} /(\varepsilon+\sigma)^{2}\right) \leq$ $\left|v_{0}\right|^{2}+C /(\varepsilon+\sigma)^{2}$ since $G / U$ is negatively curved. Thus the contribution to the angular component is bounded by

$$
\begin{aligned}
\int_{\varepsilon}^{\varepsilon+\sigma} & \left(\left|v_{0}\right|^{2}+C /(\varepsilon+\sigma)^{2}\right) d \phi r d r \\
& \leq(\varepsilon+\sigma) d \phi \sigma\left|v_{0}\right|^{2}+C \sigma d \phi /(\varepsilon+\sigma) .
\end{aligned}
$$


Now $v_{0}$ is the angular component of $\theta$ on the circle $S_{\varepsilon}$. Therefore the total contribution to the energy from both intervals is bounded by

$$
\left(L^{2}(\varepsilon+\sigma) / \sigma+(\varepsilon+\sigma) \sigma\left|\theta_{0}\right|^{2}+C|\log (\varepsilon+\sigma)|+C\right) d \phi .
$$

In other words, the total energy is bounded by

$$
\frac{L^{2}(\varepsilon+\sigma)}{\sigma}+C|\log (\varepsilon+\sigma)|+\frac{\sigma(\varepsilon+\sigma)}{\varepsilon} \int_{S_{\varepsilon}}|\theta|^{2}+C .
$$

The constant may be absorbed into $C|\log (\varepsilon+\sigma)|$ since $\varepsilon$ and $\sigma$ will be small. The condition that the metric has polynomial growth means that $L \leq C|\log (\varepsilon)|$ for some $C$. Thus we get the estimate

$$
\int_{D(\varepsilon, 1)}|\theta|^{2} \leq C \frac{\varepsilon+\sigma}{\sigma}|\log (\varepsilon)|+\frac{\sigma(\varepsilon+\sigma)}{\varepsilon} \int_{S_{\varepsilon}}|\theta|^{2} .
$$

Setting $\sigma=\varepsilon$, we get

$$
\int_{D(\varepsilon, 1)}|\theta|^{2} \leq C|\log (\varepsilon)|+2 \varepsilon \int_{S_{\varepsilon}}|\theta|^{2} .
$$

Choose a small number $\nu$, and notice that

$$
\int_{D(0,1)} r^{\nu}|\theta|^{2}=\nu \int_{0}^{1} \varepsilon^{\nu-1} d \varepsilon \int_{D(\varepsilon, 1)}|\theta|^{2}
$$

Then we get the estimate

$$
\int_{D(0,1)} r^{\nu}|\theta|^{2} \leq C \nu \int_{0}^{1}|\log \varepsilon| \varepsilon^{\nu-1} d \varepsilon+2 \nu \int_{0}^{1} \varepsilon^{\nu} \int_{S_{\varepsilon}}|\theta|^{2} .
$$

But notice that the last term is equal to $2 \nu \int_{D(0,1)} r^{\nu}|\theta|^{2}$, so we may subtract this on the other side to get

$$
(1-2 \nu) \int_{D(0,1)} r^{\nu}|\theta|^{2} \leq C \nu \int_{0}^{1}|\log (\varepsilon)| \varepsilon^{\nu-1} d \varepsilon .
$$

Since $|\log \varepsilon| \leq C_{\nu} \varepsilon^{-\nu / 2}$, the integral on the right is bounded by a constant depending on $\nu$. If $\nu<1 / 2$ we may divide, so

$$
\int_{D(0,1)} r^{\nu}|\theta|^{2} \leq C_{\nu}
$$

Recall that the eigenvalues of $\theta$ are multivalued holomorphic functions. Suppose that we did not have a bound of $C / r$ for the sizes of these eigenvalues. Then at least one of the eigenvalues would be bigger than $r^{-\mu-1}$ for some $\mu>0$. (Note that by the above estimate for example, one of the eigenvalues times $z$ is an $n$-valued holomorphic function which is square-integrable, so it cannot have an essential singularity at the origin.) In particular we would get $|\theta|^{2} \geq c r^{-2 \mu-2}$. Taking $\nu=\mu$ we would get

$$
\int_{D(0,1)} r^{\nu}|\theta|^{2} \geq c \int_{0}^{1} r^{-\nu-2} r d r
$$


but the latter integral is infinite, contradicting the previous estimate. This proves that the eigenvalues of $\theta$ are bounded by $C / r$, which is the proposition.

This completes most of the analysis which we need. The main constructions of the paper involve Weitzenböck formulas. We will now give a lemma which demonstrates an important principle which will be used several times.

Lemma 2.2. Suppose $f$ is a function smooth away from the origin, which grows more slowly than the Green's function; in other words $|f / \log r| \rightarrow 0$ as $r \rightarrow 0$. Suppose $b$ is a positive function, and suppose $\Delta f \leq-b$ away from the origin. Then $\int b<\infty$, and the above estimate holds weakly over the origin.

Proof. It is shown in [19] that if $b$ is a bounded function, then the estimate holds weakly. For each $N$, let $b_{N}$ denote the truncation $b_{N}=\min (b, N)$. Then we have $\Delta f \leq-b_{N}$, and this estimate holds weakly across the origin. Let $\varphi$ be a positive test function, nonzero at the origin. Then we get

$$
\int \varphi b_{N} \leq-\int \varphi \Delta f \text {. }
$$

This holds for all $N$, so $\int \varphi b=\lim _{N \rightarrow \infty} \int \varphi b_{N}$ is bounded. Since $\varphi$ was nonzero at the origin and $b$ is a positive function, this implies that $b$ is integrable. It is easy to see that the estimate holds weakly.

\section{Filtered OBJects}

In this section, we will use the main estimate of the last section, in conjunction with the method of Cornalba and Griffiths [3], to construct functors from the category of harmonic bundles on $X$ with logarithmic growth of $\theta$ to some categories of coherent objects on $\bar{X}$ with filtrations at the points at infinity. The point at which we use [3] was already treated in [19], so we will just quote the relevant proposition.

Define a filtered regular Higgs bundle to be an algebraic Higgs bundle $(E, \theta)$ on $X$, together with filtrations of $j_{*}^{s} E$ by coherent subsheaves

$$
j_{*}^{s} E=\bigcup_{\alpha \in \mathbf{R}} E_{\alpha, s}
$$

for each point $s \in S=\bar{X}-X$. Here $j^{s}: X \rightarrow X \cup\{s\}$ is the inclusion. The axioms are as follows (at a point $s \in S$ to which we need not refer further). First, $E_{\alpha} \subset E_{\beta}$ whenever $\alpha \geq \beta$, and $E_{\alpha}=\bigcap_{\beta<\alpha} E_{\beta}$. Next, if $z$ is a local coordinate at $s$, then $E_{\alpha+1}=z E_{\alpha}$. Finally,

$$
\theta: E_{\alpha} \rightarrow E_{\alpha} \otimes \Omega \frac{1}{X}(\log s) .
$$

Here $\Omega \frac{1}{X}(\log s)$ is the sheaf of logarithmic differentials at $s$, those generated by $d z / z$ where $z$ is a coordinate vanishing at $s$.

Similarly, define a filtered regular $\mathscr{D}_{X}$-module to be an algebraic locally free sheaf $V$ on $X$, with a connection

$$
\nabla: V \rightarrow V \otimes \Omega_{X}^{1}
$$


and filtrations of $j_{*}^{s} V$ by coherent subsheaves

$$
j_{*}^{s} V=\bigcup_{\alpha \in \mathbf{R}} V_{\alpha, s}
$$

for each point $s \in S$. The first axioms for the filtrations are the same as above, so $V_{\alpha}=\bigcap_{\beta<\alpha} V_{\beta}$, and $V_{\alpha+1}=z V_{\alpha}$. The regularity condition is

$$
\nabla: V_{\alpha} \rightarrow V_{\alpha} \otimes \Omega \frac{1}{\bar{X}}(\log s) \text {. }
$$

In other words, the operation $z \frac{\partial}{\partial z}$ preserves the $V_{\alpha}$.

More generally, define a filtered vector bundle to be a locally free sheaf $E$ together with filtrations $E_{\alpha, s}$ of $j_{*}^{s} E$ which satisfy the axioms common to the two situations above. Then a filtered regular Higgs bundle is a filtered vector bundle together with a $\theta$ satisfying a regularity condition, and a filtered regular $\mathscr{D}_{X}$-module is a filtered vector bundle together with a connection $\nabla$ satisfying a regularity condition.

Recall the following construction from $[19, \S 10]$, which we will denote by $\Xi$ (the notation of [19] was ' $a$ '). If $E$ is a holomorphic vector bundle on $X$ with a metric $K$, then define the sheaves $\Xi(E)_{\alpha}$ on $X \cup\{s\}$ as follows. The germs of sections of $\Xi(E)_{\alpha}$ at $s$ are the sections $e$ of $E$ in a punctured neighborhood of $s$, which satisfy the growth conditions $|e|_{K} \leq C r^{\alpha-\varepsilon}$ for every $\varepsilon>0$. For simplicity of notation we will sometimes denote $\Xi(E)_{\alpha}$ just by $E_{\alpha}$.

Consider the following restricted class of metrized holomorphic bundles on $X$. Say that $(E, K)$ is acceptable if the curvature $R_{K}$ of the metric connection of the metric $K$ satisfies a bound near every point $s \in S$, of the form

$$
\left|R_{K}\right| \leq f+\frac{C}{r^{2}(\log r)^{2}}
$$

for $f \in L^{p}$; here $r$ is the radius from the point $s$. The following proposition is proved (although not stated precisely this way) in $[19, \S 10]$, using the theory of Cornalba and Griffiths [3].

Proposition 3.1. If $E$ is in the class of acceptable bundles on $X$, then $\Xi(E)$ is a filtered vector bundle (i.e. the $\Xi(E)_{\alpha}$ are coherent). Furthermore, this construction on the class of acceptable bundles is compatible with the operations of taking determinants, duals, and tensor products.

Remark. The compatibility with taking the dual is the key point, meaning that the filtration accurately reflects the growth properties of the metric. The definition of the dual of a filtered vector bundle is that $\lambda \in\left(E^{*}\right)_{\alpha}$ if and only if $\lambda: E_{\beta} \rightarrow\left(\mathscr{O}_{X}\right)_{\alpha+\beta}$ for all $\beta$. The definition of dual implied by $[19$, p. 192,1 . $15]$ is incorrect; that line should read " $a\left(L^{*}\right)_{-\alpha}=(a L)_{-\alpha}^{*}$ " with the right side referring to the definition of dual described above.

Theorem 2. If $\left(E, D, D^{\prime \prime}, K\right)$ is a tame harmonic bundle on $X$ then the metrized bundle $E$, with either of the holomorphic structures $\bar{\partial}$ or $d^{\prime \prime}$, is acceptable. Furthermore, the endomorphism-valued one-form $\theta=D^{\prime \prime}-\bar{\partial}$ and 
the connection $\nabla=D$ satisfy the regularity conditions, making $(\Xi(E, \bar{\partial}), \theta)$ into a filtered regular Higgs bundle, and $\left(\Xi\left(E, d^{\prime \prime}\right), \nabla\right)$ into a filtered regular $\mathscr{D}_{X}$-module. These constructions are compatible with determinants, duals, and tensor products.

Proof. By the main estimate, we have $|\theta \bar{\theta}+\bar{\theta} \theta|$ bounded by $C / r^{2}(\log r)^{2}$. But $\theta \bar{\theta}+\bar{\theta} \theta$ is equal to the curvature of the connection $\partial+\bar{\partial}$, so $(E, K)$ is acceptable with respect to the holomorphic structure of $\bar{\partial}$. On the other hand, the metric connection for the structure $d^{\prime \prime}$ is $\delta^{\prime}+d^{\prime \prime}$. This differs from the flat connection $d^{\prime}+d^{\prime \prime}$ by $\delta^{\prime}-\delta^{\prime \prime}=2 \theta$. Thus the curvature of the connection $\delta^{\prime}+d^{\prime \prime}$ is equal to $2 d^{\prime \prime}(\theta)$. But $d^{\prime \prime}=\bar{\partial}+\bar{\theta}$ and $\bar{\partial}(\theta)=0$, so again the curvature of the metric connection $\delta^{\prime}+d^{\prime \prime}$ is $2(\theta \bar{\theta}+\bar{\theta} \theta)$. Thus $(E, K)$ is acceptable with respect to the holomorphic structure $d^{\prime \prime}$. The estimate on $|\theta|$ implies that $\theta: E_{\alpha} \rightarrow E_{\alpha} \otimes \Omega_{\bar{X}}(\log s)$, so $(\Xi(E, \bar{\partial}), \theta)$ is a filtered regular Higgs bundle.

Similarly, suppose $e$ is a section of $E$ such that $d^{\prime \prime}(e)=0$, and such that $|e| \leq r^{\alpha-\varepsilon}$. We would like to show that $|\nabla e| \leq r^{\alpha-2 \varepsilon-1}$. It suffices to consider the case when $|e| \leq r^{\varepsilon}$, and we want to show that $|\nabla(e)| \leq r^{-1-\varepsilon}$. Note that $\delta^{\prime}+d^{\prime \prime}$ is a connection compatible with the unitary structure. Let $R_{\delta^{\prime}+d^{\prime \prime}}$ denote the curvature. If $d^{\prime \prime}(e)=0$ we have the equation

$$
\Delta|e|^{2}=-\left|\delta^{\prime}(e)\right|^{2}+\operatorname{Re}\left(e, R_{\delta^{\prime}+d^{\prime \prime}} e\right) .
$$

On the other hand, $R_{\delta^{\prime}+d^{\prime \prime}}$ is a multiple of $\theta \bar{\theta}+\bar{\theta} \theta$, so it is integrable, and in fact we have

$$
\left|\operatorname{Re}\left(e, R_{\delta^{\prime}+d^{\prime \prime}} e\right)\right| \leq C / r^{2}(\log r)^{2} .
$$

This in turn is bounded by $\Delta(C \log |\log r|)$. Thus

$$
\Delta\left(|e|^{2}-C \log |\log r|\right) \leq-\left|\delta^{\prime}(e)\right|^{2} .
$$

By Lemma $2.2,\left|\delta^{\prime}(e)\right|^{2}$ is integrable and the inequality holds weakly across the puncture. On the other hand, $\nabla(e)=\delta^{\prime}(e)+2 \theta(e)$, and we know that $2 r^{\varepsilon} \theta(e)$ is in $L^{2}$ for any $\varepsilon$, by our main estimate for $|\theta|$. Therefore $r^{\varepsilon} \nabla(e)$ is in $L^{2}$ for any $\varepsilon$. We may trivialize $E$ in such a way that the filtration $E_{\alpha}$ consists of algebraic subbundles, so that $K$ grows polynomially, and see that $\nabla(e)$ is meromorphic. Let $\alpha$ be the biggest such that $\nabla(e) \in E_{\alpha}$. Then $|\nabla(e)| \geq r^{\alpha+\varepsilon}$. The condition that $\nabla(e)$ is in $L^{2}$ implies that $\alpha^{\prime} \geq-1$. This is what we are trying to prove, so it completes the theorem.

Remark. The constructions $\Xi$ are functors. We will define a morphism of tame harmonic bundles to be a map preserving the operators $D$ and $D^{\prime \prime}$, and uniformly bounded with respect to the metric. It is clear that this gives rise to maps of filtered bundles which preserve the filtrations, and also that it preserves $\theta$ or $\nabla$.

Finally we will treat a variant of the notion of filtered $\mathscr{D}_{X}$-module. It should be noted that the following discussion, while somewhat lengthy, does not have 
anything to do with the analytic details of harmonic bundles. It is included in order to provide a more intuitive topological interpretation of filtered regular $\mathscr{D}_{X}$-modules.

First, some notation. If $s$ is a point in $\bar{X}-X$, then we may consider rays $\rho$ going toward $s$. If $L$ is a local system of rank $n$, then the stalk $L_{\rho}$ is a vector space of rank $n$, isomorphic to the fiber of $L$ at any point along the ray. As the ray moves around the point, these vector spaces fit together in a locally constant way. In particular, there is a monodromy transformation $\mu_{s}$ induced on the $L_{\rho}$ by the process of continuation once around $s$ (counterclockwise). Define a filtered local system to be a locally constant sheaf $L$ of $\mathbf{C}$-vector spaces on $X$, together with the data of a filtration of the stalk

$$
L_{\rho}=\bigcup_{\alpha} L_{\rho, \alpha}
$$

(indexed by real numbers $\alpha$ ), for each ray $\rho$ approaching a point $s \in \bar{X}-X$, which is locally constant as the ray moves around $s$ (in particular, the filtration is preserved by the monodromy transformation $\mu_{s}$ ). Alternatively, one can let $U$ be a small punctured disk around $s$, and gather together the $L_{\rho, \alpha}$ for all rays $\rho$ to form a filtration of $\left.L\right|_{U}$ by subsystems $L_{U, \alpha}$. This is the same data. Note that the notion of a filtered local system is a purely topological concept.

We will define an equivalence of categories between filtered local systems and filtered regular $\mathscr{D}_{X}$-modules. Note that the question is local at a singularity. Define a functor $\Phi$ from filtered local systems to filtered regular $\mathscr{D}_{X}$-modules as follows. If $L$ is a filtered local system, then $\Phi(L)$ is the associated analytic $\mathscr{D}_{X}$-module, with the following filtration of the stalk. Let $M$ denote a logarithm of the monodromy matrix, divided by $-2 \pi i$, obtained by choosing logarithms of each of the eigenvalues. Henceforth, by "logarithm of the monodromy" we will mean a matrix $M$ normalized this way, so that the monodromy itself is $e^{-2 \pi i M}$. For any element $l$ of the stalk of $L$ over the ray $\rho$, we obtain a holomorphic section $e^{M \log z} l$ of $\Phi(L)$, where a principal determination of $\log z$ is taken at the ray $\rho$. The sheaf $\Phi(L)_{\alpha}$ is defined to be the sheaf generated by all sections of the above form, for all choices of $l \in L_{\beta}$ and all choices of $M$ with real part of the eigenvalues greater than $\alpha-\beta$. Since the monodromy preserves the filtration, so does its logarithm $M$, and

$$
\nabla e^{M \log z} l=e^{M \log z} M l \frac{d z}{z},
$$

so $\nabla$ preserves the filtration as required. Thus $\Phi(L)$ is a filtered regular $\mathscr{D}_{X^{-}}$ module.

Lemma 3.2. The functor $\Phi$ is an equivalence of categories which is compatible with direct sums, determinants, duals, and tensor products.

Proof. Denote $\Phi(L)$ by $E$. First of all, we can decompose $L=\bigoplus L^{\xi}$ by eigenvalues of the monodromy. This gives a decomposition $E \oplus E^{\xi}$. For any choice of logarithm $M$, we have $M=\bigoplus M^{\xi}$, and the eigenvalue of $M^{\xi}$ is 
$a+b i$, where $\xi=\exp (-2 \pi i a+2 \pi b)$. Note that the choice of $a$ is only well defined up to adding an integer. Since the filtration $L_{\beta}$ is compatible with the monodromy, it is compatible with the decomposition according to eigenvalues: $L_{\beta}=\bigoplus L_{\beta}^{\xi}$. Also, note that

$$
e^{\oplus M^{\xi} \log z} \sum l^{\xi}=\sum e^{M^{\xi} \log z} l^{\xi} .
$$

The filtration of $E$ generated by the elements on the right side is the direct sum of the filtrations of $E^{\xi}$ generated by the elements $e^{M^{\xi} \log z} l^{\xi}$. Note that in order for $M$ to run over all possible choices, we must have the $M^{\xi}$ exactly run over all possible choices.

Therefore the functor $\Phi$ is compatible with this decomposition according to eigenvalues. Thus we may now restrict our attention to local systems $L$ with one particular eigenvalue $\xi$, and suppress this superscript. In this case there is a better description of the filtration of $E$. Choose a logarithm $a+b i$ of $\xi$, and let $M$ denote the resulting logarithm of the monodromy. Define a vector subspace of $E$ :

$$
H=e^{M \log z} L
$$

with filtration

$$
H_{\gamma}=e^{M \log z} L_{\gamma-a} .
$$

Then $E_{\alpha}$ is the subsheaf of $E$ generated by the $t^{k} H_{\alpha-k}$ for all $k$. We may now prove that this construction is compatible with tensor products. Suppose $L^{\prime}$ and $L^{\prime \prime}$ are local systems with eigenvalues $\xi^{\prime}$ and $\xi^{\prime \prime}$, logarithms $a^{\prime}+b^{\prime} i$ and $a^{\prime \prime}+b^{\prime \prime} i$, and resulting logarithms of monodromy $M^{\prime}$ and $M^{\prime \prime}$. Then the eigenvalue of $L=L^{\prime} \otimes L^{\prime \prime}$ is $\xi=\xi^{\prime} \xi^{\prime \prime} \otimes e^{M^{\prime \prime} \log z} \otimes e^{M^{\prime \prime} \log z}$, so we may take its logarithm to be $a+b i=\left(a^{\prime}+a^{\prime \prime}\right)+\left(b^{\prime}+b^{\prime \prime}\right) i$. Then $M=M^{\prime} \otimes 1+1 \otimes M^{\prime \prime}$. Note that $e^{M \log z}=e^{M^{\prime} \log z} \otimes e^{M^{\prime \prime} \log z}$, so

$$
H=e^{M \log z} L=e^{M^{\prime} \log z} L^{\prime} \otimes e^{M^{\prime \prime} \log z} L^{\prime \prime}=H^{\prime} \otimes H^{\prime \prime} .
$$

The filtration of $H$ is the tensor product of the filtrations of $H^{\prime}$ and $H^{\prime \prime}$; in other words

$$
H_{\gamma}=\sum_{\delta} H_{\delta}^{\prime} \otimes H_{\gamma-\delta}^{\prime \prime} .
$$

(Note that by definition the same is true of the filtration of $L$.) Finally, this implies that $E_{\alpha}$ is the subsheaf generated by

$$
\sum_{k+\mu+\nu \geq \alpha} t^{k} H_{\mu}^{\prime} \otimes H_{\nu}^{\prime \prime}=\sum_{m+n+\mu+\nu \geq \alpha}\left(t^{m} H_{\mu}^{\prime}\right) \otimes\left(t^{n} H_{\nu}^{\prime \prime}\right)
$$

so

$$
E_{\alpha}=\sum_{\alpha^{\prime}+\alpha^{\prime \prime} \geq \alpha} E_{\alpha^{\prime}}^{\prime} \otimes E_{\alpha^{\prime \prime}}^{\prime \prime}
$$


This proves that our construction is compatible with tensor products of systems with a single eigenvalue. Since it is compatible with the decomposition according to eigenvalues, we have shown that the functor $\Phi$ is compatible with tensor products.

In particular this implies that it is compatible with determinants. To show that it is compatible with duals, it suffices to treat the case of a local system of rank one. Let $l$ denote the unit section. Let $\beta$ be the level of the filtration. Note that the logarithm of the monodromy is just $M=a+b i$. The section $h=e^{M \log z} l$ lies at level $a+\beta$ in the filtration of $E$. In the dual bundle with unit section $l^{*}$, the level of the filtration is $-\beta$. The logarithm of the monodromy is $-M$. The dual section to $h$ is

$$
h^{*}=e^{-M \log z} l^{*},
$$

which occurs in level $-a-\beta$ of the filtration of $E$. Thus the dual of $L$ induces the dual filtration on the dual of $E$.

We have now shown that the functor $\Phi$ is compatible with tensor products, determinants, and duals. In order to show that it is fully faithful, use the existence of internal Hom's-we have to show that $\Phi$ induces an isomorphism of global sections. Note that a global section of $L$ means a locally constant section which is contained in $L_{0}$, and a global section of $E$ means a flat section which is contained in $E_{0}$. If $l$ is a flat holomorphic section, then it is contained in the component where the eigenvalue of the monodromy is zero. Choose $M$ to have eigenvalue zero, and then $M l=0$, so $e^{M \log z} l=l$ is in $E_{\beta}$ if and only if $l$ is in $L_{\beta}$. Thus the space of flat sections which are in $L_{0}$ is the same as the space of flat sections which are in $E_{0}$, so $\Phi$ is fully faithful.

Finally we must show that $\Phi$ is essentially surjective. To make everything more precise, we work over an open neighborhood of the origin, and allow sections which are analytic on this open set, while remaining meromorphic at the origin. We also denote by $E$ the space of such sections of $E$. Fix a parameter $z$ at the origin. Then if $E$ is a regular singular $\mathscr{D}_{X}$-module, we obtain finite-dimensional vector subspaces $E^{u}$, the generalized eigenspaces of $\nabla$ with eigenvalues $u \frac{d z}{z}$. It is almost true that $E=\oplus E^{u}$; really, this direct sum is dense in the adic topology defined by order of vanishing at the origin. Or, the sections of $E$ are the convergent infinite sums of sections of $E^{u}$ for large $u$. In any case we will abuse notation and write $E$ as the direct sum. Since the filtration $E_{\alpha}$ is compatible with the operator $\nabla$, the filtration must be compatible with the direct sum decomposition by eigenvalues; in other words $E_{\alpha}=\bigoplus E_{\alpha}^{u}$. Now we may define $\mathscr{E}_{\beta}$ to be the sub- $\mathscr{D}_{X}$-module generated by the elements of $E_{\beta+a}^{a+b i}$, and in fact

$$
\mathscr{E}_{\beta}=\bigoplus_{a+b i} E_{\beta+a}^{a+b i}
$$

in our topological sense. Note that multiplication by the parameter $z$ takes $E^{a+b i}$ isomorphically to $E^{a+1+b i}$, and $E_{\alpha}$ to $E_{\alpha+1}$, so $\mathscr{E}_{\beta}$ is preserved by 
multiplication by $z$. It is clearly preserved by $\nabla$, so it is a sub- $\mathscr{D}_{X}$-module. This gives our filtered local system: $L$ is the local system of flat sections of $E$, and $L_{\beta}$ is the subsystem of flat sections of $\mathscr{E}_{\beta}$. One checks that this gives the inverse construction to the functor $\Phi$. Note that if $M$ is the logarithm of the monodromy with eigenvalues including $a+b i$, then one obtains elements $l$ of $L$ by setting

$$
l=e^{-M \log z} v
$$

for $v \in E^{a+b i}$. These span the subsystem of $L$ corresponding to the eigenvalue $\exp (-2 \pi i a+2 \pi b)$ of the monodromy. And if $v$ is at level $\beta+a$ then $l$ will be at level $\beta$. Having constructed the inverse to the construction $\Phi$, we are done with the lemma.

Remark. The inverse may be characterized as follows. If $E$ is a filtered regular $\mathscr{D}_{X}$-module, then the corresponding filtration on the local system is defined by: $L_{\beta}$ consists of all elements $l$ such that $e^{M \log z} l \in E_{\alpha}$ whenever the real parts of the eigenvalues of $M$ are at least $\alpha-\beta$. If $M$ is fixed and the real parts of the eigenvalues are all equal to $\xi$, then $l \in L_{\beta}$ if and only if $e^{M \log z} l \in E_{\beta+\xi}$.

A metric on a local system is a metric on the associated $C^{\infty}$ vector bundle over $X$. Given a metric $K$ on a local system $L$, we obtain a filtration as follows. $L_{\rho, \alpha}$ consists of horizontal sections $y$ of $L$ along the ray $\rho$ which satisfy $|y|_{K} \leq C r^{\alpha-\varepsilon}$ for any $\varepsilon>0$. Note that on $X$, the notions of local system and of analytic $\mathscr{D}_{X}$-modules are equivalent. In particular, we may speak of the pseudo-curvature $G_{K}$ of a metric $K$. We will state the following proposition without proof for the moment. The proof will be given at the end of $\S 5$.

Proposition 3.3. If $L$ is a local system with a tame harmonic metric $K$, the metric induces filtrations on $L$ and the corresponding $\mathscr{D}_{X}$-module $E$. The filtration on $L$ induced by the metric is equal to the filtration obtained in the above way from the filtration of $E$. In particular, it is preserved by the monodromy, and the dual metric induces the dual filtration on the dual local system and similarly for determinants and tensor products.

As a result of this proposition, we get a functor $\Xi$ from tame harmonic bundles to filtered local systems, sending $(E, K)$ to the local system of flat sections, together with the filtration defined by the metric $K$. This is compatible with the functor $\Xi$ to filtered regular $\mathscr{D}_{X}$-modules, via the equivalence $\Phi$ established above. When we need to distinguish between the various functors $\Xi$, they will be labeled $\Xi_{B}, \Xi_{D o l}$, and $\Xi_{D R}$, for the functors from tame harmonic bundles to filtered local systems, filtered regular Higgs bundles, and filtered regular $\mathscr{D}_{X}$-modules respectively. Thus, $\Xi_{D R}=\Phi \circ \Xi_{B}$.

In the next section, we will show that the functors $\Xi_{D o l}$ and $\Xi_{D R}$ (and hence $\Xi_{B}$ ) are fully faithful. In the subsequent section we will identify the images by some stability conditions. 


\section{SECTIONS AND MORPHISMS}

In this section we will see how the spaces of sections of bundles behave under the correspondence established in the last section, and we will use this to show that the functors $\Xi$ are fully faithful (where the morphisms of harmonic bundles are understood to be bounded morphisms).

In order to obtain the necessary estimates, let us first consider some simple Weitzenböck formulas. Suppose $E$ is a bundle with a metric $K$, and with operators $D, D^{c}, D^{\prime \prime}$, and $D^{\prime}$ related by the metric in the usual way. Let $F_{K}=D^{2}$ and $G_{K}=\left(D^{\prime \prime}\right)^{2}$. Write $D^{\prime \prime}=\bar{\partial}+\theta, D=d^{\prime}+d^{\prime \prime}$, and so forth as in $\S 1$. Some of the preliminary formulas obtained in the process of proving the following lemma will themselves be useful later on.

Lemma 4.1. Suppose $\left(E, K, D, D^{\prime \prime}\right)$ is a boundle with operators related by the metric as above. Suppose that $e$ is a section with $D(e)=0$. Then

$$
\Delta \log |e|^{2} \leq 2\left|\Lambda F_{K}-2 \Lambda G_{K}\right| \text {. }
$$

If $e$ is a section with $D^{\prime \prime}(e)=0$, then

$$
\Delta \log |e|^{2} \leq 2\left|\Lambda F_{K}-\Lambda G_{K}-\Lambda \bar{G}_{K}\right|
$$

Proof. Suppose first that $e$ is a section with $D(e)=0$. Then $d^{\prime \prime}(e)=0$ and $d^{\prime}(e)=0$, so

$$
\begin{aligned}
\partial \bar{\partial}|e|^{2} & =\partial\left(e, \delta^{\prime} e\right) \\
& =\left(\delta^{\prime}(e), \delta^{\prime}(e)\right)+\left(e, d^{\prime \prime} \delta^{\prime}(e)\right) \\
& =\left(\delta^{\prime}(e), \delta^{\prime}(e)\right)+\left(e,\left(d^{\prime \prime} \delta^{\prime}+\delta^{\prime} d^{\prime \prime}\right) e\right)
\end{aligned}
$$

For the last term, note that $\delta^{\prime}=d^{\prime}-2 \theta$. Therefore

$$
\begin{aligned}
d^{\prime \prime} \delta^{\prime}+\delta^{\prime} d^{\prime \prime} & =F_{K}-2 d^{\prime \prime}(\theta)=F_{K}-2 \bar{\partial}(\theta)-2 \bar{\theta} \theta-2 \theta \bar{\theta} \\
& =F_{K}-2 G_{K}-2(\bar{\theta} \theta+\theta \bar{\theta})
\end{aligned}
$$

Thus the second term becomes

$$
\left(e,\left(F_{K}-2 G_{K}\right) e\right)-2(\theta e, \theta e)-2(\bar{\theta} e, \bar{\theta} e) .
$$

Now on the other hand, $\delta^{\prime}(e)=-2 \theta(e)$ since $d^{\prime}(e)=0$. Therefore the first term is $4(\theta e, \theta e)$. Altogether we get

$$
\partial \bar{\partial}|e|^{2}=\left(e,\left(F_{K}-2 G_{K}\right) e\right)+2(\theta e, \theta e)-2(\bar{\theta} e, \bar{\theta} e) .
$$

Recall that $\Delta^{\prime \prime}=-\sqrt{-1} \Lambda \partial \bar{\partial}$. On the other hand,

whereas

$$
\sqrt{-1} \Lambda(\theta e, \theta e)=|\theta e|^{2}
$$

$$
\sqrt{-1} \Lambda(\bar{\theta} e, \bar{\theta} e)=-|\theta e|^{2}
$$

because $\theta e$ is of type $(1,0)$ and $\bar{\theta} e$ is of type $(0,1)$. Therefore we have

$$
\Delta^{\prime \prime}|e|^{2}=\left(e, \sqrt{-1} \Lambda\left(F_{K}-2 G_{K}\right) e\right)-2|\theta e|^{2}-2|\bar{\theta} e|^{2} .
$$

This is our preliminary Weitzenböck formula. 
We need to turn it into an estimate for $\Delta^{\prime \prime} \log |e|^{2}$. Upon writing this out it becomes

$$
\Delta^{\prime \prime} \log |e|^{2}=\frac{\Delta^{\prime \prime}|e|^{2}}{|e|^{2}}+\sqrt{-1} \Lambda \frac{\partial|e|^{2} \bar{\partial}|e|^{2}}{|e|^{4}}
$$

The last term is

$$
\sqrt{-1} \Lambda \frac{\left(\delta^{\prime} e, e\right)\left(e, \delta^{\prime} e\right)}{|e|^{4}}=\frac{\left|\left(\delta^{\prime} e, e\right)\right|^{2}}{|e|^{4}} .
$$

On the other hand, since $\delta^{\prime} e=2 \theta e$ we may write

$$
\left|\left(\delta^{\prime} e, e\right)\right|^{2}=4|(\theta e, e)|^{2}=2|(\theta e, e)|^{2}+2|(\bar{\theta} e, e)|^{2}
$$

which in turn is bounded by $|e|^{2}\left(2|\theta e|^{2}+2|\bar{\theta} e|^{2}\right)$. Putting these together with our previous version of the Weitzenböck formula, we get

$$
\Delta^{\prime \prime} \log |e|^{2} \leq \frac{\left(e, \sqrt{-1} \Lambda\left(F_{K}-2 G_{K}\right) e\right)}{|e|^{2}},
$$

or in other words (changing to $\Delta=2 \Delta^{\prime \prime}$ ), we get the first half of the lemma.

To prove the second half of the lemma, proceed in a similar fashion. Suppose that $e$ is a section with $D^{\prime \prime}(e)=0$. Then $\bar{\partial}(e)=0$ and $\theta(e)=0$, so

$$
\begin{aligned}
\partial \bar{\partial}|e|^{2} & =\partial(e, \partial e) \\
& =(\partial(e), \partial(e))+(e, \bar{\partial} \partial(e)) \\
& =(\partial(e), \partial(e))+(e,(\partial \bar{\partial}+\bar{\partial} \partial) e) .
\end{aligned}
$$

For the last term, note that

$$
\partial \bar{\partial}+\bar{\partial} \partial=F_{K}-G_{K}-\bar{G}_{K}-\theta \bar{\theta}-\bar{\theta} \theta
$$

Thus the last term becomes

$$
\left(e,\left(F_{K}-G_{K}-\bar{G}_{K}\right) e\right)-(\bar{\theta} e, \bar{\theta} e)
$$

so

$$
\Delta^{\prime \prime}|e|^{2}=\left(e, \sqrt{-1} \Lambda\left(F_{K}-G_{K}-\bar{G}_{K}\right) e\right)-\left|D^{\prime}(e)\right|^{2},
$$

the preliminary Weitzenböck formula for the second half. Now turn to the logarithm of $|e|^{2}$. Again,

$$
\Delta^{\prime \prime} \log |e|^{2}=\frac{\Delta^{\prime \prime}|e|^{2}}{|e|^{2}}+\sqrt{-1} \Lambda \frac{\partial|e|^{2} \bar{\partial}|e|^{2}}{|e|^{4}}
$$

The second term is $\left.\left.|\partial| e\right|^{2}\right|^{2} /|e|^{4}$ which is equal to $|(\partial e, e)|^{2} /|e|^{4}$. This in turn is less than or equal to $|\partial e|^{2} /|e|^{2}$, which is certainly bounded by $\left|D^{\prime}(e)\right|^{2} /|e|^{2}$. Thus we get

$$
\Delta^{\prime \prime} \log |e|^{2} \leq \frac{\left(e, \sqrt{-1} \Lambda\left(F_{K}-G_{K}-\bar{G}_{K}\right) e\right)}{|e|^{2}} \leq\left|F_{K}-G_{K}-\bar{G}_{K}\right|,
$$

as desired. 
Corollary 4.2. Suppose $\left(E, K, D, D^{\prime \prime}\right)$ is an object with the two operators related by the metric. Suppose that $\left|F_{K}\right|+\left|G_{K}\right| \in L^{p}$ for some $p>1$. Suppose that $e$ is a section with $D(e)=0$ or $D^{\prime \prime}(e)=0$, and with $|e| \leq C r^{-\varepsilon}$ for all $\varepsilon>0$. Then $|e|$ is bounded.

Proof. By the Weitzenböck formulas, we have in either case

$$
\Delta \log |e|^{2} \leq h
$$

for a positive function $h$ which is $L^{p}$ for $p>1$. We may find a bounded function $u$ with $\Delta(u)=h$. Thus $\Delta\left(\log |e|^{2}-u\right) \leq 0$. This estimate holds weakly across the origin, due to the estimate $|e| \leq C r^{-\varepsilon}$ for all $\varepsilon$ (see Lemma 2.2). Furthermore, we may assume $\log |e|^{2}-u \leq 0$ on the boundary of the unit disk. It follows that $\log |e|^{2} \leq u$ inside the unit disk.

Corollary 4.3. Given a bundle $E$ with either a flat connection $D$ or a Higgs operator $D^{\prime \prime}$, and two metrics $H$ and $K$, suppose that the curvatures $G_{H}$ and $G_{K}$, or $F_{H}$ and $F_{K}$, respectively, are in $L^{p}$. Suppose furthermore that the metrics $H$ and $K$ give rise to the same filtration $\left\{E_{\alpha}\right\}$ on $j_{*}(E)$, and give rise to the dual of this filtration on $j_{*}\left(E^{*}\right)$. Then $H$ and $K$ are mutually bounded.

Proof. Apply the previous corollary to the identity map $e$ from $(E, H)$ to $(E, K)$, considered as a section of $E^{*} \otimes E$. Then do the same for the map in the other direction. The condition that the filtrations are all the same means that $|e| \leq C r^{-\varepsilon}$ for all $\varepsilon>0$ so we may apply the previous corollary to conclude that $e$ is bounded.

Theorem 3. The functors $\Xi$ from the category of tame harmonic bundles on $X-$ where the maps are maps preserving the operators and bounded with respect to the metrics-to the categories of filtered regular Higgs bundles and filtered regular $\mathscr{D}_{X}$-modules, are fully faithful.

Proof. Recall that the constructions $\Xi$ commute with duals and tensor products. A map of filtered regular objects from $\Xi(E)$ to $\Xi\left(E^{\prime}\right)$ is a section $f$ of the bundle $E^{*} \otimes E^{\prime}$, killed by $D^{\prime \prime}$ or $D$ respectively, and whose norm satisfies $|f| \leq C r^{-\varepsilon}$ for all $\varepsilon>0$. By the above results, $f$ is bounded. We must show that if $D^{\prime \prime}(f)=0$ then $D^{\prime}(f)=0$, or that if $D(f)=0$ then $\theta(f)=0$ and $\bar{\theta}(f)=0$ in the second case (this will imply $\left.D^{\prime \prime}(f)=\left(d^{\prime \prime}-\bar{\theta}+\theta\right)(f)=0\right)$. But note that in both cases, both curvatures $F_{K}$ and $G_{K}$ vanish. Therefore our preliminary Weitzenböck formulas from the proof of Lemma 4.1 read

$$
\Delta|f|^{2}=-2\left|D^{\prime} f\right|^{2}
$$

in the first case, and

$$
\Delta|f|^{2}=-4|\theta f|^{2}-4|\bar{\theta} f|^{2}
$$

in the second. These hold over $X$. However, since we know that $|f|$ is bounded, they hold weakly over all of $\bar{X}$. But the integral over $\bar{X}$ of the Laplacian of any distribution is zero. Thus in the first case, $D^{\prime}(f)=0$, while the second case, $\theta(f)=0$ and $\bar{\theta}(f)=0$. This proves the theorem. 


\section{LOCAL STUDY}

In this section, we will construct model metrics locally at the singularities. In $\S 6$ these will be used to construct harmonic metrics with the desired local behavior. We will also obtain estimates for the growth of the norms of holomorphic or flat sections, taken up in more detail in $\S 7$.

First, we deal with line bundles. Suppose $E$ is a filtered line bundle with $E_{\alpha}=\mathscr{O}_{\bar{X}}$, and $E_{\alpha+\varepsilon} \neq E_{\alpha}$ (in other words, $\alpha$ is where the jump occurs). The standard metric on $E$ is the metric $S$ with $|1|_{S}=r^{\alpha}$. Note that this makes reference to the chosen isomorphism $E_{\alpha} \cong \mathscr{O}_{\bar{X}}$, as well as to a chosen local coordinate $z$ at the puncture. The metric connection of this metric is flat, since $\Delta \log r=0$. Let $\partial_{0}$ denote the $(1,0)$ part of the standard connection on $\mathscr{O}_{\bar{X}}$, and let $\partial$ denote the $(1,0)$ part of the metric connection of $S$. Note that the function $h_{S}$ describing the difference between the metric $S$ and the usual one is actually $h_{S}=r^{2 \alpha}=z^{\alpha} \bar{z}^{\alpha}$. Thus

$$
\partial=\partial_{0}+\alpha \frac{d z}{z} \text {. }
$$

If $L$ is endowed with a one-form $\theta=a \frac{d z}{z}$ then $\bar{\theta}=\bar{a} \frac{d \bar{z}}{\bar{z}}$. We have

$$
\begin{aligned}
D=D^{\prime}+D^{\prime \prime} & =d^{\prime}+d^{\prime \prime}, \\
D^{\prime \prime}=\bar{\partial}+a \frac{d z}{z}, & D^{\prime}=\bar{\partial}_{0}+\alpha \frac{d z}{z}+\bar{a} \frac{d \bar{z}}{\bar{z}}, \\
d^{\prime \prime}=\bar{\partial}+\bar{a} \frac{d \bar{z}}{\bar{z}}, & d^{\prime}=\partial_{0}+(a+\alpha) \frac{d z}{z} .
\end{aligned}
$$

We will define a new section $v$ of $E$ which is holomorphic with respect to the operator $d^{\prime \prime}$. Set $v=r^{-2 \bar{a}} \cdot 1$. Since $a$ may be imaginary, this should be taken as $\exp (-2 \bar{a} \log r)$ which is well defined since, $r$ being real, we may take the real determination of $\log r$. Note that $\bar{\partial} v=-\bar{a} \frac{d \bar{z}}{\bar{z}} v$, so $d^{\prime \prime}(v)=0$. Use $v$ to give a trivialization of the holomorphic bundle $V=\left(E, d^{\prime \prime}\right)$. With respect to this trivialization, the unit section (which we will still call $v$ to avoid confusion) has norm

$$
|v|_{S}=r^{\alpha-(a+\bar{a})}
$$

Therefore the jump in the filtration of $V$ occurs at $\alpha-(a+\bar{a})$ in contrast to the fact that the jump in the filtration of $E$ was at $\alpha$. We have $d^{\prime}(v)=$ $(-\bar{a}+a+\alpha) \frac{d z}{z} v$; in other words with respect to the trivialization given by the unit section $v$, the connection form for $\nabla$ is $(a-\bar{a}+\alpha) d z / z$. Setting $a=b+c i$ we may summarize this situation in the following table. The third column describes the filtered local system associated to $(V, \nabla)$ as discussed in $\S 3$.

We now turn to the other type of models, which are the variations of Hodge structure corresponding to representations of $S L_{2}$ considered by Schmid [18, $\S 5]$. Let $W$ be the two-dimensional variation of Hodge structure corresponding to the standard representation of $S L_{2}$. It is a harmonic bundle over the 


\begin{tabular}{|c|c|c|c|}
\hline & $(E, \theta)$ & $(V, \nabla)$ & $L$ \\
\hline jump & $\alpha$ & $\alpha-2 b$ & $-2 b$ \\
\hline residue & $b+c i$ & $\alpha+2 c i$ & $\exp (-2 \pi i \alpha+4 \pi c)$ \\
\hline
\end{tabular}

punctured disk. We have $W=W^{1,0} \oplus W^{0,1}$. These bundles have holomorphic structures which we denote $\bar{\partial}$. Let $w^{1,0}$ and $w^{0,1}$ denote the unit holomorphic sections in these bundles. We have $\theta: W^{1,0} \rightarrow W^{0,1} \otimes\langle d z / z\rangle$ defined by

$$
\theta\left(w^{1,0}\right)=\frac{1}{2} w^{0,1} \frac{d z}{z}, \quad \theta\left(w^{0,1}\right)=0 .
$$

The metric $T$ of $W$ is defined by $\left|w^{1,0}\right|_{T}=|\log r|^{1 / 2},\left|w^{0,1}\right|_{T}=|\log r|^{-1 / 2}$, and $w^{1,0} \perp w^{0,1}$. This gives a harmonic metric.

The associated $\mathscr{D}_{X}$-module may be described as follows. The section $w^{1,0}$ is killed by $\bar{\theta}$, and hence is $d^{\prime \prime}$-holomorphic. On the other hand,

$$
\bar{\theta}\left(w^{0,1}\right)=\frac{d \bar{z}}{2 \bar{z}|\log r|^{2}} w^{1,0},
$$

so the section

$$
v^{0,1} \stackrel{\text { def }}{=} w^{0,1}-w^{1,0} /|\log r|
$$

is $d^{\prime \prime}$-holomorphic. Thus the sections $w^{1,0}$ and $v^{0,1}$ form our basis. We have

$$
\nabla\left(w^{1,0}\right)=\frac{1}{2} \frac{d z}{z} v^{0,1}, \quad \nabla\left(v^{0,1}\right)=0 .
$$

The norms are $\left|w^{1,0}\right|=|\log r|^{1 / 2}$ and $\left|v^{0,1}\right|=\sqrt{2}|\log r|^{-1 / 2}$. Bear in mind that these sections are not perpendicular: $\left(w^{1,0}, v^{0,1}\right)=-1$. On the other hand, if one sets them to be perpendicular with the same norms, an equivalent metric is obtained.

The associated flat bundle has sections $v^{0,1}$ and $u^{1,0}=w^{1,0}-\frac{1}{2} v^{0,1} \log z$. In particular, the monodromy matrix is a unipotent $2 \times 2$ matrix, with the invariant subspace spanned by $v^{0,1}$. The norms of the flat sections are $\left|v^{1,0}\right|=$ $\sqrt{2}|\log r|^{-1 / 2}$, and on any sector, $\left|u^{1,0}\right| \sim|\log r|^{1 / 2} / \sqrt{2}$.

Suppose $(E, \theta)$ is a filtered regular Higgs bundle. Then for a puncture $y \in S$ we will define a pair $(V, N)=\operatorname{Res}_{y}(E, \theta)$ consisting of a collection of vector spaces $V_{\alpha}$ and a collection of endomorphisms $N_{\alpha}$ of $V_{\alpha}$. Also part of the data are canonical identifications $\left(V_{\alpha}, N_{\alpha}\right) \cong\left(V_{\alpha+1}, N_{\alpha+1}\right)$. Thus in fact the data consist of the objects for $0 \leq \alpha<1$. $V_{\alpha}$ is equal to the vector space of sections of the skyscraper sheaf $\operatorname{Gr}_{\alpha}(E)=E_{\alpha} / E_{\alpha+\varepsilon} . N$ is equal to the residue of $\operatorname{Gr}(\theta)$.

Similarly if $(E, \nabla)$ is a filtered regular $\mathscr{D}_{X}$-module, we define $(V, N)=$ $\operatorname{Res}(E, \nabla)$. The $V_{\alpha}$ are defined the same way. To define $N_{\alpha}$ as the residue of $\nabla$, note that $\nabla: E_{\alpha} \rightarrow E_{\alpha \alpha} \otimes \dot{O}_{X}\langle d z / z\rangle$. The graded version of this map, 
$\mathrm{Gr}_{\alpha} \nabla: \mathrm{Gr}_{\alpha} E \rightarrow \mathrm{Gr}_{\alpha} E \otimes \mathrm{C}\langle d z / z\rangle$ is linear, and the residue evaluation $d z / z \mapsto$ 1 is canonical.

From the two kinds of systems constructed above, we may build up other systems. By taking symmetric powers of $W$, we get systems where the residue $N$ of $\theta$ (or the residue of $\nabla$ ) is a nilpotent matrix consisting of one Jordan block of arbitrary size. By taking the tensor product with rank one objects of the form described above, we may insure that the residue of $\theta$ or $\nabla$ has any eigenvalue, and that the jump in the filtration occurs at any $\alpha$. Then we may take direct sums of these objects. The conclusion is that given any vector space $V=\bigoplus_{0 \leq \alpha<1} V_{\alpha}$ with an endomorphism $N$ compatible with the decomposition, there exists a harmonic bundle $E$ such that $\operatorname{Res}(a E, \theta)=$ $(V, N)$ or $\operatorname{Res}(a E, \nabla)=(V, N)$.

Theorem 4. Given any filtered regular Higgs bundle, $\mathscr{D}_{X}$-module, or local system, there is a metric $K$ whose curvature $\left(F_{K}\right.$ or $G_{K}$, respectively) is in $L^{p}$ for some $p>1$, which induces the desired filtration and the dual of the desired filtration on the dual object. Furthermore we may assume that the curvature $R_{K}$ of the metric connection is acceptable.

Proof. Let $(V, N)=\operatorname{Res}(E)$ as defined above (in either the Higgs or $\mathscr{D}_{X^{-}}$ module case). Then there is a harmonic bundle $E_{1}$ with $\operatorname{Res}\left(E_{1}\right)=(V, N)$. We may choose an isomorphism of filtered bundles $E_{1} \cong E$ which is the identity on the graded quotient $V$. Hence $\operatorname{Res}(\theta)=\operatorname{Res}\left(\theta_{1}\right)\left(\right.$ or $\left.\operatorname{Res}(\nabla)=\operatorname{Res}\left(\nabla_{1}\right)\right)$. Let $A=\theta-\theta_{1}$ in the first case, and let $A=\nabla-\nabla_{1}$ in the second case. Then in either case we have

$$
A: E_{\alpha} \rightarrow E_{\alpha+\varepsilon} \otimes \Omega_{\bar{X}}^{1}(\log S) .
$$

Let $K$ denote the image of the harmonic metric of $E_{1}$, via $\phi$. Then $K$ is a harmonic metric with respect to $\theta_{1}$ or $\nabla_{1}$. We claim that the curvature $F_{K}$ or $G_{K}$ of $K$ with respect to $\theta$ or $\nabla$ is in $L^{p}$ as desired. Note that in any event $K$ is an acceptable metric on $E$, because the holomorphic structures of $E$ and $E_{1}$ are the same (so $R_{K}$ is the same for $E$ and $E_{1}$ ). We have $|A| \leq C r^{\varepsilon-1}$ for some $\varepsilon>0$.

Now in the first case, $\theta=\theta_{1}+A$. We know that $R_{K}+\theta_{1} \bar{\theta}_{1}+\bar{\theta}_{1} \theta_{1}=0$, as this is the curvature of $E_{1}$. Therefore

$$
\begin{aligned}
F_{K} & =R_{K}+\theta \bar{\theta}+\bar{\theta} \theta \\
& =\theta_{1} \bar{A}+\bar{A} \theta_{1}+A \bar{\theta}_{1}+\bar{\theta}_{1} A+A \bar{A}+\bar{A} A
\end{aligned}
$$

is bounded by $C r^{\varepsilon / 2-2}$, since $\left|\theta_{1}\right| \leq C r^{-1-\varepsilon / 4}$. Thus $F_{K}$ is in $L^{p}$ for some $p>1$.

In the second case, $\nabla=\nabla_{1}+A$. Hence $d^{\prime}=d_{1}^{\prime}+A$, whereas $\delta^{\prime}=\delta_{1}^{\prime}$ since $d^{\prime \prime}=d_{1}^{\prime \prime}$. Thus $\theta=\theta_{1}+A / 2$. But we know that $-2 G_{K, 1}=R_{K}+2 \theta_{1} \bar{\theta}_{1}+$ $2 \bar{\theta}_{2} \theta_{1}=0$. Therefore

$$
\begin{aligned}
-2 G_{K} & =R_{K}+2 \theta \bar{\theta}+2 \bar{\theta} \theta \\
& =\theta_{1} \bar{A}+\bar{A} \theta_{1}+A \bar{\theta}_{1}+\bar{\theta}_{1} A+A \bar{A}+\bar{A} A
\end{aligned}
$$


is again bounded by $C r^{\varepsilon / 2-2}$. Thus $G_{K}$ is in $L^{p}$ for some $p>1$.

To complete the proof for the case of a local system, apply the statement to the corresponding $\mathscr{D}_{X}$-module to get a standard metric. We will show that if $(E, \nabla)$ is a filtered regular $\mathscr{D}_{X}$-module, with associated filtered local system $L$, and if $K$ is a suitable standard metric on $E$ (and hence on $L$ ), then it induces the filtration of $L$. Suppose $l$ is an element of $L$ in $L_{\beta}$ but not in $L_{\beta+\varepsilon}$. We would first like to show that $|l| \leq C r^{\beta-\varepsilon}$. We may decompose according to eigenvalues of the monodromy (before choosing our standard metric on $E$ ) so we may assume that there is only one eigenvalue of the monodromy. Let $M$ be a logarithm of the monodromy (divided by $-2 \pi i$ as usual), with eigenvalue $a+b i$. Let $h=e^{M \log z} l$, so $h \in E_{\beta+a}$ but $h$ is not in $E_{\beta+a+\varepsilon}$. By construction of the standard metric on $E$, we have $|h| \sim r^{\beta+a}$. Since $l=e^{-M \log z} h$ we have $|l| \leq C r^{\beta-\varepsilon}$ for all $\varepsilon>0$.

On the other hand, the association between $E$ and the filtered local system $L$ is compatible with taking duals. We may find a dual section $\lambda$ in $\left(L^{*}\right)_{-\beta}$ such that $\lambda(l)=1$. But we know as above that $|\lambda| \leq C r^{-\beta-\varepsilon}$. Thus

$$
1 \leq|\lambda||l| \leq C r^{-\beta-\varepsilon}|l| \text {. }
$$

and so $|l| \geq c r^{\beta+\varepsilon}$. Thus the metric on $L$ induces the filtration $L_{\beta}$, completing the proof.

Proof of Proposition 3.3. If $K$ is a tame harmonic metric, then it induces a filtration on the $\mathscr{D}_{X}$-module $E$. If we choose a standard metric $H$ for this filtration, then by Corollary 4.3, $H$ and $K$ are mutually bounded. Since $H$ induces the appropriate filtration on $L$ as shown above, the same is true of $K$.

Remark. We can apply this method more generally. If we start with a tame harmonic bundle and form the associated filtered regular Higgs bundle or filtered regular $\mathscr{D}_{X}$-module, then we can use the construction of this section to put on a model metric giving the same filtration. By Corollary 4.3, the harmonic metric is bounded with respect to the model metric. This observation provides estimates on the growth of the harmonic metric, which will be explored in more detail in $\S 7$.

\section{Stability AND EXISTENCE Results}

In this section we will identify the images of the functors $\Xi$ from the category of tame harmonic bundles to the categories of regular filtered Higgs bundles, and regular filtered $\mathscr{D}_{X}$-modules (and hence also, filtered local systems). In previous sections, we have shown how a tame harmonic bundle gives a filtered regular Higgs bundle, and a filtered regular $\mathscr{D}_{X}$-module. The purpose of this section is to go back in the other direction, starting from a filtered regular Higgs bundle or $\mathscr{D}_{X}$-module, to construct a tame harmonic bundle. This works if and only if the filtered regular object satisfies a stability condition. We will just state the main existence theorem, because this direction has already been dealt 
with in [19] for the case of Higgs bundles. A gap in the exposition is that we have not given the proof of the other half of the main existence theorem for $\mathscr{D}_{X}$-modules. This has been done in the compact case $[2,7]$, and the arguments to treat the noncompact case may be taken from [19].

First we define notions of degree of a metrized bundle and of a filtered bundle. We will prove that they coincide in the case when the filtration comes from an acceptable metric.

If $(E, K)$ is a holomorphic vector bundle with a metric, then its first Chern form is $c_{1}(E, K)=\operatorname{Tr}\left(R_{K}\right)$, where $R_{K}$ is the curvature of the metric connection. Define the analytic degree to be

$$
\operatorname{deg}(E, K)=\int_{\bar{X}} \operatorname{Tr}\left(R_{K}\right) .
$$

This may be a well-defined number, $+\infty$ or $-\infty$, or not convergent. This notion of degree is compatible with taking the determinant of the bundle-then one gets a line bundle whose curvature is the trace of the original curvature. If we ignore convergence problems, then it is compatible with all of the usual formulas for degrees of tensor products, direct sums, and duals.

If $\left(E,\left\{E_{\alpha, x}\right\}\right)$ is a filtered bundle on $X$, then define its algebraic degree as

$$
\operatorname{deg}\left(E,\left\{E_{\alpha, x}\right\}\right)=\operatorname{deg}\left(E_{0}\right)+\sum_{x} \sum_{0 \leq \alpha<1} \alpha \operatorname{rk}\left(\mathrm{Gr}_{\alpha}\left(E_{x}\right)\right) .
$$

This notion of degree is compatible with all of the usual formulas for degrees of determinants, tensor products, duals, and direct sums.

Lemma 6.1. If $E$ is a holomorphic bundle with an acceptable metric, then the degree is convergent. If $\left\{E_{\alpha, x}\right\}$ denotes the filtration corresponding to $K$, then

$$
\operatorname{deg}(E, K)=\operatorname{deg}\left(E,\left\{E_{\alpha, x}\right\}\right) .
$$

Proof. If $(E, K)$ is acceptable, then in particular its curvature $R_{K}$ is in $L^{1}$ so the degree is absolutely convergent. We may go to the line bundle $\operatorname{det}(E)$. Since the construction $\Xi$ is compatible with taking determinants, as are both notions of degree, it suffices to prove that $\operatorname{deg}(L, K)=\operatorname{deg}\left(L,\left\{L_{\alpha, x}\right\}\right)$ for a line bundle $L$. We may further tensor with a line bundle on $\bar{X}$, so we may assume that $L_{0}=\mathscr{O}_{\bar{X}}$. Let $\alpha_{x}$ denote the jumps in the filtrations, with $0 \leq \alpha_{x}<1$. Then

$$
\operatorname{deg}\left(L,\left\{L_{\alpha, x}\right\}\right)=\sum_{x} \alpha_{x} .
$$

On the other hand, at each point $x$ the norm of the unit section 1 is given approximately by $|1| \sim r^{\alpha_{x}}$. Now $\Delta_{X} \log |1|=R$ is an $L^{1}$ function on $X$. By the estimate for the growth of $|1|$ we get the equation of distributions on $\bar{X}$ :

$$
\Delta_{\bar{X}} \log |1|=R-\sum_{x} \alpha_{x} \delta_{x}
$$


where $\delta_{x}$ is a delta-function located at $x$. The integral of $\Delta_{\bar{X}} \log |1|$ over $\bar{X}$ is equal to zero, so we get

$$
\operatorname{deg}(L, K)=\int_{X} R=\sum_{x} \alpha_{x}
$$

This proves the lemma.

Lemma 6.2. If $E$ is a holomorphic bundle with an acceptable metric $K$, and if $F \subset E$ is a holomorphic subbundle, we may restrict the metric to $F$. Then either: (1) $\operatorname{deg}(F, K)=-\infty$; or (2) $\operatorname{deg}(F, K)$ is finite, and $F$ can be extended to a meromorphic subsheaf of $\Xi(E)$. In the latter case, $F$ becomes a filtered bundle, and we have $\operatorname{deg}\left(F,\left\{F_{\alpha}\right\}\right)=\operatorname{deg}(F, K)$.

Proof. See [19, Lemmas 3.2, 10.5, and 10.6, and Corollary 10.7]. The basic point is the Chern-Weil formula. Let $\pi$ denote the orthogonal projection from $E$ to $F$ using the metric $K$. Recall that $R_{K}$ denotes the curvature on $E$. The following is the classical statement that curvature decreases in holomorphic subbundles:

$$
\operatorname{deg}(F, K)=\sqrt{-1} \int_{X} \operatorname{Tr}\left(\pi \Lambda R_{K}\right)-\int_{X}|\bar{\partial} \pi|^{2} .
$$

Thus if $R_{K}$ is integrable, then $\operatorname{deg}(F, K)$ will be either $-\infty$ or finite. If it is not $-\infty$, then $F$ is an $L_{1}^{2}$ subsystem. In this case one shows that it is algebraic, and the degree is the same as the degree calculated by the induced filtration (cf. [19, Lemmas 10.5 and 10.6]). The proof of the fact that the algebraic and analytic degrees are the same in the above mentioned Lemma 10.5 does not quite apply here, but it is easily modified to work. The main point used there is that if $e$ is a section of $E$ which lies in $E_{\alpha}$ but not in $E_{\alpha+\varepsilon}$ for any $\varepsilon>0$, then $|e|_{K} \sim r^{\alpha}$ in the sense that

$$
\left.|\log | e\right|_{K}-\log r^{\alpha}|\leq \varepsilon| \log r \mid
$$

for any $\varepsilon$ (and small $r$ depending on $\varepsilon$ ). Note that in [19] there was a better estimate of $\log |\log r|$, but the above would do just as well in applying the remark following [19, Proposition 2.2]. To prove the estimate in our case (using only the assumption that $K$ is an acceptable metric), note that by definition of $E_{\alpha}$, we have

$$
\log |e|_{K}-\log r^{\alpha} \leq \varepsilon|\log r|
$$

for any $\varepsilon$. On the other hand, we may choose $\lambda$ to be a section of the dual $E^{*}$ such that $\lambda \in E_{-\alpha}^{*}$ and $\lambda(e)=1$. The fact that $K$ induces the dual filtration on the dual implies that

$$
\log |\lambda|_{K^{*}}+\log r^{\alpha} \leq \varepsilon|\log r|
$$

for any $\varepsilon$. On the oiher hand, $1 \leq|\lambda|_{K^{*}}|e|_{K}$, so

$$
\log |e|_{K} \geq-\log |\lambda|_{K^{*}} \geq \log r^{\alpha}-\varepsilon|\log r| \text {. }
$$

Together these give

$$
\left.|\log | e\right|_{K}-\log r^{\alpha}|\leq \varepsilon| \log r \mid
$$


as desired. The remainder of the proof of [19, Lemma 10.5] carries through, bearing in mind the remarks during and following Proposition 2.2 there (Lemma 2.2 here).

We give two sets of definitions of stability for Higgs bundles and $\mathscr{D}_{X}$-modules. The first definitions are analytic, and depend on the choice of a metric $K$. The second definitions are algebraic, and depend on the choices of filtrations at the points at infinity. In the case when the filtrations come from an appropriate metric, it will be a consequence of the previous lemma that the two definitions of stability coincide.

First some discussion of hypotheses is in order. The notion of analytic degree depends only on having a curvature which is in $L^{1}$. This can either be the curvature of the holomorphic bundle, or one of the curvatures $F_{K}$ or $G_{K}$ as appropriate (cf. the remarks below). The notion of analytic stability depends on the extra structure $\theta$ or $\nabla$, for one considers subobjects which respect this extra structure. However, it is not necessary to assume that the $\theta$ or $\nabla$ have any particular properties related to the metric. The situation is similar for algebraic degree and stability. For the notion of algebraic stability, it is not necessary to assume that $\theta$ or $\nabla$ respect the filtrations in any way. In fact, it is not even necessary to assume that $\theta$ or $\nabla$ are meromorphic. Of course, in practice $\theta$ or $\nabla$ will be regular with respect to the filtration.

Suppose $\left(E, D^{\prime \prime}\right)$ is a Higgs bundle on $X$. Fix a metric $K$, and assume that the curvature $F_{K}$ is in $L^{1}$. Say that $\left(E, D^{\prime \prime}, K\right)$ is analytically stable (resp. semistable) if for all subbundles $M \subset E$ with $D^{\prime \prime} M \subset M$ (i.e. $M$ is holomorphic and preserved by $\theta$ ), we have

$$
\frac{\operatorname{deg}(M, K)}{\operatorname{rk}(M)}<(\leq) \frac{\operatorname{deg}(E, K)}{\operatorname{rk}(E)} .
$$

Note that this makes sense, because the degree of $M$ is either $-\infty$ (so the condition is automatic), or else a finite number.

Remark. In calculating $\operatorname{deg}(E, K)$, we may use either the curvature $F_{K}$, or the curvature $R_{K}$ of the metric connection $\partial_{K}+\bar{\partial}$. This is because they differ by $\theta \bar{\theta}+\bar{\theta} \theta$, and $\operatorname{Tr}(\theta \bar{\theta}+\bar{\theta} \theta)=0$. The same goes for any subobject. Note that if $F_{K}$ is used, then the extra term in the Chern-Weil formula becomes $\int_{X}\left|D^{\prime \prime} \pi\right|^{2}$ instead of $\int_{X}|\bar{\partial} \pi|^{2}$.

Suppose $(V, D)$ is a $\mathscr{D}_{X}$-module on $X$. Fix a metric $K$, and assurne that either $G_{K}$ or $R_{K}$ is in $L^{1}$. Define the degree of $V$ using the curvature $R_{K}$ (and similarly for any subobject). Say that $(V, D, K)$ is analytically stable (resp. semistable) if for all sub- $\mathscr{D}_{X}$-modules $W \subset V$ (i.e. $W$ is holomorphic and preserved by $\nabla$ ), we have

$$
\frac{\operatorname{deg}(W, K)}{\operatorname{rk}(W)}<(\leq) \frac{\operatorname{deg}(V, K)}{\operatorname{rk}(V)} .
$$

Remark. As before, in calculating the degree $\operatorname{deg}(V, K)$ we may use the pseudocurvature $-2 G_{K}$ instead of the curvature $R_{K}$ of the metric connection. This is 
because

$$
\begin{aligned}
-2 G_{K} & =-2 \bar{\partial}(\theta)=-\left(d^{\prime \prime}+\delta^{\prime \prime}\right)\left(d^{\prime}-\delta^{\prime}\right) / 2 \\
& =-d^{\prime}\left(\delta^{\prime \prime}\right) / 2+d^{\prime \prime}\left(\delta^{\prime}\right) / 2,
\end{aligned}
$$

whereas $R_{K}=d^{\prime \prime}\left(\delta^{\prime}\right)$. Now $2 \theta \bar{\theta}+2 \bar{\theta} \theta$ is equal to $\left(d^{\prime \prime}-\delta^{\prime \prime}\right)\left(d^{\prime}-\delta^{\prime}\right) / 2=$ $-d^{\prime}\left(\delta^{\prime \prime}\right) / 2-d^{\prime \prime}\left(\delta^{\prime}\right) / 2$. Therefore we have

$$
R_{K}=-2 G_{K}-2 \theta \bar{\theta}-2 \bar{\theta} \theta .
$$

But $\operatorname{Tr}(\theta \bar{\theta}+\bar{\theta} \theta)=0$, so when calculating degrees, the integrands $\operatorname{Tr}\left(R_{K}\right)$ and $\operatorname{Tr}\left(-2 G_{K}\right)$ are the same.

We should explicitly note the form of the Chern-Weil formula if the curvature $-2 G_{K}$ is integrated to obtain the degree of a subsystem. Suppose $W \subset V$ is a sub- $\mathscr{D}_{X}$-module, and let $\pi$ denote the orthogonal projection onto $W$. The condition that $W$ is a subsystem means that $(1-\pi) D(\pi)=0$. We have

$$
D_{W}=\pi D \pi=D \pi=\pi D-\pi D(\pi)
$$

and

$$
D_{W}^{c}=\pi D^{c} \pi=\pi D^{c}-\pi D^{c}(\pi) .
$$

Here $D^{c}=D^{\prime \prime}-D^{\prime}$. Hence

$$
4 G_{W}=D_{W} D_{W}^{c}+D_{W}^{c} D_{W}=\pi D \pi D^{c} \pi+\pi D^{c} \pi D \pi .
$$

The first term is equal to $\pi D D^{c} \pi+\pi D(\pi) D^{c} \pi$. Now note that $\pi D(\pi)=D(\pi)$ and $D(\pi) \pi=0$. Therefore the first term becomes $\pi D D^{c} \pi+D(\pi) D^{c}(\pi)$. The second term is just $\pi D^{c} D \pi$. Thus

$$
4 G_{W}=4 \pi G \pi+D(\pi) D^{c}(\pi),
$$

hence

$$
4 \sqrt{-1} \operatorname{Tr}\left(\Lambda G_{W}\right)=4 \sqrt{-1} \operatorname{Tr}(\pi \Lambda G)+\sqrt{-1} \Lambda \operatorname{Tr}\left(D(\pi) D^{c}(\pi)\right) .
$$

The last term is equal to $\sqrt{-1} \Lambda \operatorname{Tr}\left(d^{\prime}(\pi) \delta^{\prime \prime}(\pi)\right)-\sqrt{-1} \Lambda \operatorname{Tr}\left(d^{\prime \prime}(\pi) \delta^{\prime}(\pi)\right)$. By the Kähler identities relating $d^{\prime \prime}$ and $\delta^{\prime}$ and vice-versa, this last term is equal to $|D(\pi)|^{2}$. Thus

$$
\sqrt{-1} \Lambda \operatorname{Tr}\left(-2 G_{K, W}\right)=\sqrt{-1} \Lambda \operatorname{Tr}\left(-2 \pi G_{K, V}\right)-|D(\pi)|^{2} / 2 .
$$

So the Chern-Weil formula written in terms of $-2 G_{K}$ is

$$
\operatorname{deg}(V, K)=\sqrt{-1} \int_{X} \Lambda \operatorname{Tr}\left(-2 \pi G_{K}\right)-\frac{1}{2} \int_{X}|D(\pi)|^{2} .
$$

Suppose we are given a filtered regular Higgs bundle $\left(E, \theta,\left\{E_{\alpha}\right\}\right)$. Then we say that it is algebraically stable (resp. semistable) if for all algebraic subbundles $F \subset E$ preserved by $\theta$,

$$
\frac{\operatorname{deg}\left(F,\left\{F_{\alpha}\right\}\right)}{\operatorname{rk}(F)}<(\leq) \frac{\operatorname{deg}\left(E,\left\{E_{\alpha}\right\}\right)}{\operatorname{rk}(E)}
$$


Here, $\left\{F_{\alpha}\right\}$ denotes the filtration induced on $j_{*}(F)$ by the given filtration on $j_{*}(V)$.

Similarly, if we are given a filtered regular $\mathscr{D}_{X}$-module $\left(V, \nabla,\left\{V_{\alpha}\right\}\right)$, we say that it is algebraically stable (resp. semistable) if for all algebraic subbundles $F \subset E$ preserved by $\theta$,

$$
\frac{\operatorname{deg}\left(W,\left\{W_{\alpha}\right\}\right)}{\operatorname{rk}(W)}<(\leq) \frac{\operatorname{deg}\left(V,\left\{V_{\alpha}\right\}\right)}{\operatorname{rk}(V)}
$$

Here, $\left\{W_{\alpha}\right\}$ denotes the filtration induced on $j_{*}(W)$ by the given filtration on $j_{*}(V)$.

Lemma 6.3. If $(E, K)$ is a Higgs bundle or $\mathscr{D}_{X}$-module with metric such that $R_{K}$ is acceptable, let $\left(E,\left\{E_{\alpha}\right\}\right)$ denote the corresponding filtered object constructed in $\S 3$. Then analytic stability (resp. semistability) of $(E, K)$ is equivalent to algebraic stability (resp. semistability) of $\left(E,\left\{E_{\alpha}\right\}\right)$.

Proof. This follows immediately from Lemma 6.2. As noted previously, one needs no conditions on the behavior of $\theta$ or $\nabla$ at infinity, measured by the metric or the filtrations.

Theorem 5. Suppose $\left(E, D^{\prime \prime}, D, K\right)$ is an irreducible tame harmonic bundle. Then the resulting (metrized or filtered) Higgs bundle or $\mathscr{D}_{X}$-module is (analytically or algebraically) stable of degree zero. If $\left(E, D^{\prime \prime}, D, K\right)$ is any tame harmonic bundle, then it is a direct sum of irreducible ones.

Proof. The degrees are zero since $G_{K}=0$ and $F_{K}=0$. By Lemma 6.3 it suffices to prove analytic stability. Suppose $M \subset E$ is a subbundle preserved by $D\left(\right.$ resp. $\left.D^{\prime \prime}\right)$. Suppose that $\operatorname{deg}(M, K, D)=0$ (resp. $\left.\operatorname{deg}\left(M, K, D^{\prime \prime}\right)=0\right)$. Let $\pi$ denote the orthogonal projection to $M$. Then in the Chern-Weil formula for calculations with the pseudo-curvature $G$ (resp. the curvature $F$ ), the first term is identically zero since $G_{K}=0$ (and $F_{K}=0$ ). Thus we have

$$
0=\operatorname{deg}(M, K, D)=-\frac{1}{2} \int_{X}|D(\pi)|^{2}
$$

(resp. $0=\operatorname{deg}\left(M, K, D^{\prime \prime}\right)=-\int_{X}\left|D^{\prime \prime}(\pi)\right|^{2}$ ) and hence $D(\pi)=0$ (resp. $D^{\prime \prime}(\pi)=0$ ). But $\pi$ is bounded, so also $D^{\prime \prime}(\pi)=0$ (resp. $D(\pi)=0$ ) (see the proof of Theorem 3 ). Therefore in either case, $\pi$ gives an orthogonal decomposition of $\left(E, D, D^{\prime \prime}, K\right)$ as a direct sum of two harmonic bundles. If $E$ is irreducible, then no such subsystem $M$ can exist, and in any case an inductive application of this procedure expresses any harmonic bundle $E$ as a direct sum of irreducible and hence stable factors.

Now we may state the main existence result.

Theorem 6. 1. Suppose $E$ is an analytic Higgs bundle on $X$ with a metric $K$. Assume that the curvature $F_{K}$ is in $L^{p}(p>1)$ and that $(E, K)$ is analytically stable, with analytic degree zero. Then there is a harmonic metric $H$ which is bounded with respect to $K$. 
2. Suppose $V$ is an analytic $\mathscr{D}_{X}$-module on $X$ with a metric $K$. Assume that the pseudo-curvature $G_{K}$ is in $L^{p}(p>1)$. Suppose that $(V, K)$ is analytically stable, with analytic degree zero. Then there is a harmonic metric $H$ which is bounded with respect to $K$.

Proof. The first part is proved in [19]. The second part is left to the reader. (Hint: The compact case is treated in Corlette's paper [2]; generalize to the noncompact case as in the first part, noting that the symbols $D^{\prime \prime}$ and $D, D^{\prime}$ and $D^{c}, F_{K}$ and $-4 G_{K}, \Delta^{\prime}$ and $\Delta^{c}$, and occasionally $\bar{\partial}$ and $d$, are basically interchangeable. All of the required positivity statements remain true, even though a few intermediate signs are different.)

Corollary 6.4. The filtered regular Higgs bundles or $\mathscr{D}_{X}$-modules, which come from tame harmonic bundles by the functors $\Xi_{D o l}$ and $\Xi_{D R}$, consist exactly of the objects which are direct sums of stable objects of degree zero.

Proof. Suppose $E$ is a stable filtered regular Higgs bundle of degree 0. By Theorem 4 there is an acceptable metric such that $F_{K}$ is in $L^{p}$ which induces the given filtration. By Lemma 6.3 , this metric is analytically stable, and by Lemma 6.1 the analytic degree is zero. By Theorem 5 there is a harmonic metric $H$ which is bounded with respect to $K$. Hence $H$ induces the same filtration. Thus our filtered regular Higgs bundle comes from a harmonic bundle. Note that tameness is a property of the Higgs bundle, and it is implied by the condition of regularity with respect to the filtration. The proof beginning with a filtered regular $\mathscr{D}_{X}$-module can be phrased identically. At the end, note that the flat sections of the $\mathscr{D}_{X}$-module grow polynomially with respect to the standard metric $K$ constructed in Theorem 4, and hence they grow polynomially with respect to the harmonic metric $H$. By Proposition 2.1, the harmonic bundle is tame.

Finally we include the notion of filtered local system. The degree of a filtered local system is defined to be the sum of the jumps in the filtrations at all points $s$, counted with multiplies.

Lemma 6.5. The degree of a filtered local system agrees with the degree of the corresponding filtered $\mathscr{D}_{X}$-module.

Proof. It suffices to look at objects of rank 1. Pick an extension of the line bundle to $\bar{X}$ which has degree zero. Then there is a connection $d$ which is smooth over $\bar{X}$, and $\nabla=d+a$ where $a$ is a scalar valued one-form with poles of order one. The sum of the eigenvalues of $\operatorname{res}(\nabla)$ must be equal to zero, by the residue theorem. The degree of the $\mathscr{D}_{X}$-module is equal to the sum of the jumps in the filtration which define the particular extension we have chosen, since the degree of this extension is otherwise zero. On the other hand, the jumps in the filtration of the local system are the differences between the jumps in the filtration of the line bundle, and the eigenvalues of $\operatorname{res}(\nabla)$. Since the sum of the residues vanishes, the sum of the jumps for the local system is the same as that for the $\mathscr{D}_{X}$-module, giving the lemma. 
Corollary 6.6. The notions of stability and semistability are preserved by the equivalence $\Phi$ between filtered local systems and filtered regular $\mathscr{D}_{X}$-modules.

Proof. We have to show that if $U \subset L$ is a subsystem of a filtered local system, with induced filtration, then the filtration of the $\mathscr{D}_{X}$-module $\Phi(U)$ is that induced by its being a submodule of $\Phi(L)$. To prove this we may decompose according to eigenvalues of the monodromy. Choose a logarithm $M$ with real part of the eigenvalue equal to $\xi$. The subsystem $U$ is preserved by $M$ (its restriction there is again the logarithm of the monodromy). Our statement follows from the characterization that $l \in L_{\beta}$ if and only if $e^{M \log z} l \in \Phi(L)_{\beta+\xi}$, and the same characterization for $U$.

Putting everything together, we get our main result.

Main Theorem. The category of tame harmonic bundles is naturally equivalent, via the functors $\Xi$, to the categories of direct sums of stable filtered regular Higgs bundles of degree zero, of direct sums of stable filtered regular $\mathscr{D}_{X}$-modules of degree zero, and of direct sums of stable filtered local systems of degree zero.

\section{Weight Filtrations}

In this section we will continue the local study near a singularity. We will introduce weight filtrations, following the ideas developed by Schmid and Deligne in the case of variations of Hodge structure. In particular, we will obtain a new proof of Schmid's norm estimates for variations of Hodge structure [18], and will extend these estimates to regular harmonic bundles.

The main point to notice is that in $\S 5$ we constructed some standard metrics on a filtered regular Higgs bundle or $\mathscr{D}_{X}$-module. However, by Corollary 4.3, these standard metrics must be mutually bounded with respect to any harmonic metric with the same associated filtration. In the first part of this section we will simply give a method for describing the estimates one obtains in this way.

Recall that if $E$ is a filtered bundle, then we define $V=\operatorname{Res}(E)$ to be the associated graded vector space for the filtration at some point $s \in S$. Then we obtain an endomorphism $N=\operatorname{Res}(\theta)$ or $N=\operatorname{Res}(\nabla)$ of the graded vector space $V$. We may decompose $V_{\alpha}=\bigoplus_{a+b i} V_{\alpha, a+b i}$ according to the generalized eigenvalues $a+b i$ of $N$. Then on each piece $V_{\alpha, a+b i}$ the nilpotent endomorphism $N-a-b i$ defines a weight filtration $W_{k} V_{\alpha, a+b i}$ indexed by integers $k$ (cf. [18]). This is characterized by two properties: first, that if $N$ is a nilpotent upper triangular $2 \times 2$ matrix, then its weight filtration is $W_{-2}=0$, $W_{-1}=W_{0}=\operatorname{Im}(N)=\operatorname{Ker}(N)$, and $W_{1}$ is the whole space; and second, that the construction is compatible with direct sums and symmetric powers. See the Synopsis for a description in terms of Jordan normal form.

Suppose $E$ is a tame harmonic bundle, and let $(V, N)$ be the residue of $(\Xi(E, \bar{\partial}), \theta)$ or $\left(\Xi\left(E, d^{\prime \prime}\right), \nabla\right)$. Let $(\mathscr{V}, \theta)$ or $(\mathscr{V}, \nabla)$ be a standard object with residue $(V, N)$ and the standard metric, described in $\S 5$. Our standard metric on $E$ is obtained as the image of the standard metric, by some isomorphism of filtered bundles $\mathscr{V} \cong E$. The standard metric, and the weight 
filtration, are both obtained from symmetric powers of the two-dimensional nilpotent object. Thus we may characterize the weight filtration as follows. A vector $v \in V_{\alpha, a+b i}$ is contained in $W_{k}$ if and only if, for any extension $\tilde{v}$ to a holomorphic section of $\mathscr{V}$, we have the estimate

$$
|\tilde{v}|_{\mathrm{Std}} \leq C r^{\alpha}|\log r|^{k / 2}
$$

This is because this property is preserved by symmetric powers and direct sums, and it holds for the basic standard objects ( $(5)$. By Corollary 4.3, the image of the weight filtration in $E$ may be characterized in the same way, using the harmonic metric. We may define a combined filtration $W(\alpha, k)$ of the stalk $j_{*} E_{X}$, indexed by the set of $(\alpha, k)$ in lexicographic order, so that a section $e$ is in $W(\alpha, k)$ if and only if

$$
|e|_{K} \leq C r^{\alpha}|\log r|^{k / 2}
$$

Then the residue of this filtration on $\operatorname{Gr}_{\alpha}(E)$ is equal to the weight filtration of the endomorphism $N$. This filtration is compatible with all tensor operations. It encodes the norm estimates for holomorphic sections of the Higgs bundle or $\mathscr{D}_{X}$-module associated to the tame harmonic bundle.

Next, we would like to translate this into information about norms of flat sections. Define the weight filtration of a filtered local system as follows. For each $\beta$, the quotient $\mathrm{Gr}_{\beta}(L)=L_{\beta} / L_{\beta+\varepsilon}$ is a vector space with action of the associated graded of the monodromy matrix. Decompose according to eigenvalues, and then on each generalized eigenspace form the weight filtration using the nilpotent part of the monodromy, in the same way as above. This defines a weight filtration $W_{k}$ of $\mathrm{Gr}_{\beta} L$, and we may lift this to a filtration of $L_{\beta}$. Putting these together for all $\beta$ we get a weight filtration $W(\beta, k)$ indexed by $(\beta, k)$ in lexicographic order.

Suppose that the eigenvalues of the monodromy are all the same, and choose a logarithm $M$ with eigenvalue $a+b i$. Then we contend that $l \in W(\beta, k) L$ if and only if $h=e^{M \log z} l$ is in $W(\beta+a, k) E$. To see this, note that $l \mapsto e^{M \log z} l$ gives a map from $\mathrm{Gr}_{\beta}(L)$ to $\mathrm{Gr}_{\beta+a}(E)$ (by the definition of the relation between the filtrations). Note that $\nabla e^{M \log z} l=M e^{M \log z} l d z / z$ since $l$ is a flat section. Therefore under this map, $\operatorname{res}(\nabla)$ is equal to $M$. Thus the weight filtrations are the same.

Given this fact, we obtain the norm estimates for flat sections: if $L$ is a local system with metric such that $G_{K} \in L^{p}$ for $p>1$, then

$$
l \in W(\beta, k) L \quad \text { if and only if } \quad|l| \leq C r^{\beta}|\log r|^{k / 2} .
$$

To prove this note that by Corollary 4.3 we may assume that the metric is a standard one for the $\mathscr{D}_{X}$-module. Hence we may decompose according to the eigenvalue of the monodromy, and assume that there is only one eigenvalue. Choose a logarithm $M$ (divided by $-2 \pi i$ as usual), and write $M=a+b i+N$ 
with $N$ nilpotent. If $l \in W(\beta, k) L$ then $h=e^{M \log z} l$ is in $W(\beta+a, k) E$, so by the norm estimates for $E$, we have $|h| \leq C r^{\beta+a}|\log r|^{k / 2}$. Now

$$
l=e^{-M \log z} h=z^{-a-b i}\left(1-N \log z+\frac{N^{2}}{2}(\log z)^{2}-\cdots\right) h .
$$

Note that $N^{i} h \in W(\beta+a, k-2 i) E$ (by the definition of the weight filtration), so $\left|N^{i} h\right| \leq C r^{\beta+a}|\log r|^{k / 2-i}$. Thus we get

$$
|l| \leq C r^{\beta}|\log r|^{k / 2} \text {. }
$$

Now suppose $l$ is in $W(\beta, k)$ but not $W(\beta, k-1)$. Again, everything is compatible with passage to the dual (including the constructions of the weight filtrations on $E$ and $L$ ), so we may choose a dual vector $\lambda \in W(-\beta,-k) L^{*}$ with $\lambda(l)=1$. By the same argument applied to the dual, we get $|\lambda| \leq$ $C r^{-\beta}|\log r|^{-k / 2}$, and from $1 \leq|\lambda||l|$ we get $|l| \geq c r^{\beta}|\log r|^{k / 2}$. This completes the proof of the norm estimates.

We may rephrase the estimates for the behavior of the harmonic metric on the local system in terms of the equivariant harmonic map. Fix a ray $\rho$ going out to the puncture. Let $\phi: \rho \rightarrow \mathrm{Gl}(n, \mathrm{C}) / \mathrm{U}(n)$ be the harmonic map (restricted to one copy of the ray in the universal cover). If we construct a standard metric along the ray which satisfies the same norm estimates with respect to this weight filtration, and satisfies the appropriate estimates on the dual, then it will be bounded with respect to the harmonic metric. This means that the points in the classifying space $\mathrm{Gl}(n, \mathrm{C}) / \mathrm{U}(n)$ for metrics will remain a bounded distance apart, distance being measured with an invariant metric. To construct the standard metric, choose a basis compatible with the weight filtration, and set the norms of the basis elements to be $r^{\beta}|\log r|^{k / 2}$ for appropriate $\beta$ and $k$ as described by the weight filtration. The resulting map from the ray $\rho$ into the classifying space is given by the diagonal matrix with diagonal entries $r^{\beta}|\log r|^{k / 2}$. Thus the values of the equivariant harmonic map lie within a bounded distance of the values of the map given by this diagonal matrix. Note that the matrix is diagonal in the specific basis given by the weight filtration, which may involve a change of basis from a fixed frame at the base point.

Isomorphism of the residues and weight filtrations. In the remainder, we will show that if a filtered regular Higgs bundle $E$ and a filtered regular $\mathscr{D}_{X}$-module $V$ are related by our construction (i.e. both come from a harmonic bundle), then the spaces $\operatorname{Gr}(E, s)$ and $\operatorname{Gr}(V, s)$ are isomorphic, with the structures of indexed decompositions, decompositions into eigenspaces of $\operatorname{res}(\theta)$ or $\operatorname{res}(\nabla)$, and weight filtrations (bearing in mind the change of indexing represented in the table in $\S 5$ ). We do not construct a canonical isomorphism.

I had originally hoped that the methods here would represent a simplification of the methods used by Schmid to obtain his norm estimates, and from what we know so far, one does obtain norm estimates. However, the present topic is clearly a necessary piece of information, and we will have to go through 
considerable additional difficulties to get it. Furthermore, it would obviously be beneficial to have canonical isomorphisms. To get these, it seems probable that one would have to prove an $\mathrm{Sl}_{2}$ orbit theorem for harmonic bundles. So the original optimism is unfounded-not much is gained by avoiding the $\mathrm{Sl}_{2}$ orbit theorem. The question of how to prove its analogue in the present case is an interesting problem for further study.

Let $(E, \bar{\partial}+\theta, K)$ be the filtered regular Higgs bundle with a harmonic metric, and let $\left(V, d^{\prime}+d^{\prime \prime}, K\right)$ be the filtered regular $\mathscr{D}_{X}$-module with the same metric. First we remark on the metrics. There are equivalent standard metrics $K_{0}$ on $E$ and $K_{1}$ on $V$. The metric $K_{0}$ is harmonic with respect to a Higgs operator $\theta_{0}$, which has residue equal to that of $\theta$. The metric $K_{0}$ induces a different holomorphic structure $d_{0}^{\prime \prime}=\bar{\partial}+\bar{\theta}_{0}^{K_{0}}$, giving a holomorphic bundle we call $V_{0}$. In fact, $V_{0}$ is a filtered regular $\mathscr{D}_{X}$-module, with the associated graded and residues which correctly correspond to those of $E$. What we are really trying to prove is that $\operatorname{Gr}(V, s) \cong \operatorname{Gr}\left(V_{0}, s\right)$.

There is a holomorphic frame $\left\{e_{i}\right\}$ for $E$ which is adapted to the metric $K_{0}$, in the sense that

$$
\left|e_{i}\right|=r^{a_{i}}|\log r|^{n_{i}}
$$

and $\left(e_{i}, e_{j}\right)=0$. The same is not quite true of the metric $K_{1}$ on $V$. However, there is an equivalent metric $K_{2}$ such that there is an adapted basis. To see this, it suffices to see it for the standard two-dimensional object described in $\S 5$. We have two $d^{\prime \prime}$-holomorphic sections $w^{1,0}$ and $v^{0,1}$, with $\left|w^{1,0}\right|_{K_{1}}^{2}=|\log r|$ and $\left|v^{0,1}\right|_{K_{1}}^{2}=2|\log r|^{-1}$. However, $\left(w^{1,0}, v^{0,1}\right)_{K_{1}}=-1$ (see $\left.\S 5\right)$. Define the new metric $K_{2}$ by $\left|w^{1,0}\right|_{K_{2}}^{2}=|\log r|$ and $\left|v^{0,1}\right|_{K_{2}}^{2}=|\log r|^{-1}$, but with $\left(w^{1,0}, v^{0,1}\right)_{K_{2}}=0$. Putting $u=x|\log r|^{-1 / 2} w^{1,0}+y|\log r|^{1 / 2} v^{0,1}$, we see that $|u|_{K_{1}}^{2}=|x|^{2}+2|y|^{2}-x \bar{y}-y \bar{x}$ whereas $|u|_{K_{2}}^{2}=|x|^{2}+|y|^{2}$. These norms are equivalent. Now by taking tensor products and direct sums we get our standard metric $K_{2}$. Note that if we are taking norms of things, we may use any of the metrics interchangeably, so we may for all practical purposes assume that the bases $\left\{e_{i}\right\}$ and $\left\{v_{j}\right\}$ are adapted whichever metric is being used. All of the metrics are equivalent, so we need not specify which ones are used in the discussion to follow.

Our first task is to make a preliminary effort to solve the $d^{\prime \prime}$ problem for the bundle $V$. Constants will be implicit but dropped, and we will assume that $r$ is small.

Lemma 7.1. Suppose $\eta$ is a $(0,1)$ form with coefficients in $V$, with $|\eta| \leq$ $r^{\alpha}|\log r|^{k}$. Then there is a section $H \eta$ such that $d^{\prime \prime}(H \eta)=\eta$ and such that $|H \eta| \leq r^{\alpha+1}|\log r|^{k+1} \log |\log r|$.

Proof. Choose an adapted basis $\left\{v_{j}\right\}$ with norms $r^{\alpha_{j}}|\log r|^{k_{j}}$, such that either $-2<\alpha-\alpha_{j}<-1$, or $\alpha-\alpha_{j}=-2$ and $k-k_{j} \leq-1$, or $\alpha-\alpha_{j}=-1$ and 
$k-k_{j} \geq-1$. In terms of this basis we may write

$$
\eta=\sum_{j} m_{j} v_{j}
$$

with $\left|m_{j}\right| \leq r^{\alpha-\alpha_{j}}|\log r|^{k-k_{j}}$. Let $h$ denote the usual integral operator which solves the $\bar{\partial}$ problem for scalars, namely

$$
h(m)(x)=\iint \frac{m(y)}{x-y} d \operatorname{vol}(y) .
$$

Recall that $\bar{\partial} h(m)=m$. Our assertions about this operator are as follows, supposing that $|m| \leq r^{a}|\log r|^{n}$. If $-2<a<-1$, then

$$
|h(m)| \leq r^{a+1}|\log r|^{n}
$$

if $a=-2$ and $n<-1$ or $a=-1$ and $n>-1$, then

$$
|h(m)| \leq r^{a+1}|\log r|^{n+1}
$$

and finally if $a=-1$ or $a=-2$, and $n=-1$, then

$$
|h(m)| \leq r^{a+1}|\log r|^{n+1} \log |\log r| .
$$

If we can prove these assertions, then setting

$$
H \eta=\sum_{j} h\left(m_{j}\right) v_{j}
$$

and applying the assertions term by term with $a=\alpha-\alpha_{j}$ and $n=k-k_{j}$, we will get $|H \eta| \leq r^{\alpha+1}|\log r|^{k+1} \log |\log r|$ as desired.

Before proving the assertions, we note the asymptotic behavior of some indefinite integrals involving logarithms. Namely,

$$
\int t^{u}|\log t|^{v} d t \sim C t^{u+1}|\log t|^{v}
$$

as $t \rightarrow 0$, provided that $u \neq-1$. If $u=-1$ then $t^{u} d t=d \log t$, and we get

$$
\int t^{-1}|\log t|^{v}=C|\log t|^{v+1}
$$

unless of course $v=-1$ in which case the integral is $C \log |\log t|$.

To prove the assertions, note that

$$
|h(m)(x)| \leq I(|x|) \stackrel{\text { def }}{=} \int \frac{|y|^{a}|\log | y||^{n}}{|x-y|} d \operatorname{vol}(y),
$$

the integration being taken over a disk of fixed radius $B$, say less than 1 . Divide the integral into three regions:

(i) $|y| \leq|x| / 2$,

(ii) $|x| / 2 \leq|y| \leq 2|x|$,

(iii) $|y| \geq 2|x|$. 
Denote the three integrals by $I_{(\mathrm{i})}(r), I_{(\mathrm{ii})}(r)$, and $I_{(\mathrm{iii})}(r)$, with $r=|x|$.

Region (i): We have $1 /|x-y| \leq C /|x|$, so

$$
I_{(\mathrm{i})}(r) \leq \frac{C}{r} \int_{0}^{r} s^{a+1}|\log s|^{n} d s .
$$

(The additional factor of $s$ in the integrand comes from the volume form $s d s d \theta$.) If $a>-2$ then as noted above the integral is less than $C r^{a+1}|\log r|^{n}$. If $a=-2$ but $n<-1$, it is less than $C r^{a+1}|\log r|^{n+1}$, and if $a=-2$ and $n=-1$ it is less than $C r^{a+1}|\log r|^{n+1} \log |\log r|$.

Region (ii): We have $|y| \sim|x|$, so

$$
I_{(\mathrm{ii})}(r) \sim C r^{a}|\log r|^{n} \int_{r / 2 \leq|y| \leq 2 r} \frac{d \operatorname{vol}(y)}{|x-y|}
$$

(with $|x|=r$ ). We may as well replace the region of integration by the bigger region $|x-y| \leq C r$, so the integral becomes

$$
\int_{0}^{C r} \frac{d \operatorname{vol}(x-y)}{|x-y|}=C \int_{0}^{C r} \frac{s d s}{s}=C r .
$$

Thus $I_{\text {(ii) }}(r) \leq C r^{a+1}|\log r|^{n}$.

Region (iii): We have $1 /|x-y| \leq C /|y|$, so

$$
I_{(\text {iii) }}(r) \leq \int_{r}^{B} s^{a}|\log s|^{n} d s .
$$

(In this case the additional factor of $s$ from the volume form is canceled by the factor of $|y|=s$ in the denominator.) If $a<-1$ then as noted previously the integral is less than $C r^{a+1}|\log r|^{n}$. If $a=-1$ and $n>-1$, it is less than $C r^{a+1}|\log r|^{n+1}$, and if $a=-1$ and $n=-1$ it is less than $C r^{a+1}|\log r|^{n+1} \log |\log r|$.

These together prove the assertions about the behavior of $|h(m)(x)|$, which completes the proof of the lemma.

Next we would like to show that the decomposition according to eigenvalues of $\operatorname{res}(\theta)$ is essentially the same (up to a change of indexing) as the decomposition according to eigenvalues of $\operatorname{res}(\nabla)$, to a certain order.

By choosing an appropriate holomorphic basis, we may define a $d^{\prime \prime}$-holomorphic endomorphism $\psi$ of $V$, such that if $v$ is a holomorphic section with $|v| \sim r^{\alpha}|\log r|^{k}$, then $\nabla v$ is approximately equal to $(\alpha d z / z+\psi) v$, in the sense that

$$
|\nabla v-v \alpha d z / z-\psi v| \leq r^{\alpha-1}|\log r|^{k-1} .
$$

The endomorphism $\psi$ will be semisimple with eigenvalues of the form $\beta d z / z$.

Lemma 7.2. The endomorphisms $\psi$ and $\theta$ are close in the sense that for any $\varepsilon>0$,

$$
\int|2 \theta-\psi|^{2}|\log r|^{1-\varepsilon} d \text { vol }<\infty .
$$


Proof. We will show that if $v$ is a holomorphic section with $|v| \sim r^{\alpha}|\log r|^{k}$, then

$$
\int|2 \theta(v)-\psi(v)|^{2} r^{-2 \alpha}|\log r|^{1-\varepsilon-2 k} d \mathrm{vol}<\infty .
$$

Since we may as well assume that our metric comes from a basis of such vectors $v$, this will prove the lemma. By tensoring with line bundles, we may assume that $\alpha=0$. By the definition of $\psi$ we have

$$
|\psi(v)-2 \theta(v)|^{2} \leq|\nabla(v)-2 \theta(v)|^{2}+C r^{-2}|\log r|^{2 k-2} .
$$

For the second term on the right, note that

$$
\begin{gathered}
\int\left(r^{-2}|\log r|^{2 k-2}\right)|\log r|^{1-\varepsilon-2 k} d \text { vol } \\
=C \int \frac{r d r}{r^{2}|\log r|^{1+\varepsilon}}<\infty
\end{gathered}
$$

For the first term on the right, recall that $\nabla=\delta^{\prime}+2 \theta$, where $\delta^{\prime}+d^{\prime \prime}$ is a metric compatible connection. Thus we have to show that

$$
\int\left|\delta^{\prime}(v)\right|^{2}|\log r|^{1-\varepsilon-2 k} d \text { vol }<\infty
$$

We will use the Weitzenböck formula for the connection $\delta^{\prime}+d^{\prime \prime}$ which was used in the proof of Lemma 4.1:

$$
\Delta|v|^{2} \leq-\left|\delta^{\prime}(v)\right|^{2}+C|v|^{2} r^{-2}|\log r|^{-2} .
$$

Twist this formula by $|\log r|^{1-\varepsilon-2 k}$. Note that

$$
\Delta\left(f|\log r|^{p}\right) \leq|\log r|^{p} \Delta(f)+p(p-1) f r^{-2}|\log r|^{p-2}+2 p|d f| r^{-1}|\log r|^{p-1} .
$$

Furthermore, $d|v|^{2}=\operatorname{Re}\left(v, \delta^{\prime} v\right)$ (since $d^{\prime \prime} v=0$ ), so $\left.|d| v\right|^{2}|\leq| v|| \delta^{\prime} v \mid$. Putting these together we get

$$
\begin{aligned}
\Delta\left(|v|^{2}|\log r|^{1-\varepsilon-2 k}\right) \leq & -\left|\delta^{\prime} v\right|^{2}|\log r|^{1-\varepsilon-2 k}+C|v|^{2} r^{-2}|\log r|^{-1-\varepsilon-2 k} \\
& +C|v|\left|\delta^{\prime} v\right| r^{-1}|\log r|^{-\varepsilon-2 k}
\end{aligned}
$$

On the other hand, note that

$$
|v|\left|\delta^{\prime} v\right| r^{-1}|\log r|^{-1} \leq C_{1}|v|^{2} r^{-2}|\log r|^{-2}+C_{2}\left|\delta^{\prime} v\right|^{2},
$$

where $C_{2}$ can be made small at the expense of making $C_{1}$ large. Thus we can remove the last term in the previous inequality:

$$
\Delta\left(|v|^{2}|\log r|^{1-\varepsilon-2 k}\right) \leq-\left|\delta^{\prime} v\right|^{2}|\log r|^{1-\varepsilon-2 k}+C|v|^{2} r^{-2}|\log r|^{-1-\varepsilon-2 k} .
$$

Furthermore, recall that $|v|^{2} \sim|\log r|^{2 k}$, so we get

$$
\Delta\left(|v|^{2}|\log r|^{1-\varepsilon-2 k}\right) \leq-\left|\delta^{\prime} v\right|^{2}|\log r|^{1-\varepsilon-2 k}+C r^{-2}|\log r|^{-1-\varepsilon} .
$$


The last term $C r^{-2}|\log r|^{-1-\varepsilon}$ is the Laplacian of $C|\log r|^{1-\varepsilon}$, so we may subtract that to the other side to get

$$
\Delta\left(\left(|v|^{2}|\log r|^{-2 k}+C\right)|\log r|^{1-\varepsilon}\right) \leq-\left|\delta^{\prime} v\right|^{2}|\log r|^{1-\varepsilon-2 k} .
$$

Note that $\left(|v|^{2}|\log r|^{-2 k}+C\right)$ is bounded, so the operand of the Laplacian on the left-hand side is a function which grows slower than the Green's function. It follows that the right-hand side is an $L^{1}$ function, and that the inequality holds weakly (see Lemma 2.2). The integrability of the right side is exactly the statement we needed to prove the lemma.

Lemma 7.3. We have $\left|\bar{\theta}_{0}^{K_{0}}-\bar{\theta}^{K}\right| \leq r^{-1}|\log r|^{-1}$.

Proof. Let us recall some information from $\S 2$. We have

$$
\theta=\sigma+\tau=\alpha+\beta+\tau^{0}+\tau^{+},
$$

where $\alpha$ is diagonal (in an orthonormal basis) with entries $\lambda_{i} d z / z, \beta$ is diagonal with $|\beta| \leq r^{-1+\varepsilon}$, and $\tau$ is upper triangular with $|\tau| \leq C r^{-1}|\log r|^{-1} . \tau^{0}$ is the component of $\tau$ with $(i, j)$ entries for $\lambda_{i}=\lambda_{j}$, and $\tau^{+}$is the component with $(i, j)$ entries for $\lambda_{i} \neq \lambda_{j}$. Note that $\beta$ and $\tau$ may be multivalued (with finite branching), but $\alpha, \beta+\tau^{0}$, and $\tau^{+}$are single valued. Furthermore we know that $\left|\tau^{+}\right| \leq r^{-1+\varepsilon}$. There is an endomorphism $\phi$ which is $\bar{\partial}$-holomorphic and semisimple with eigenvalues $\lambda_{i} d z / z$. The eigenspaces of $\phi$ are the sums of the generalized eigenspaces for $\theta$ for eigenvalues of residues $\lambda_{i}$. We know that $\phi=\alpha+q$, where $|q| \leq r^{-1+\varepsilon}$. Let $\tilde{\phi}$ be the matrix with the same eigenspaces as $\phi$, but with complex conjugate eigenvalues. Note that there is a polynomial $P$ with constant coefficients such that

$$
\bar{z} \tilde{\phi} / d \bar{z}=P(z \phi / d z) \text {. }
$$

The same polynomial gives $\overline{z \alpha} / d \bar{z}=P(z \alpha / d z)$. Now $|z q / d z| \leq r^{\varepsilon}$. Therefore

$$
P(z \alpha / d z+z q / d z)=P(z \alpha / d z)+R,
$$

where $|R| \leq r^{\varepsilon}$. Therefore

$$
\tilde{\phi}=\bar{\alpha}+\tilde{q}
$$

with $|\tilde{q}| \leq r^{-1+\varepsilon}$. On the other hand, $\bar{\phi}=\bar{\alpha}+\bar{q}$ and $|\bar{q}| \leq r^{-1+\varepsilon}$. Therefore

$$
|\bar{\phi}-\tilde{\phi}| \leq r^{-1+\varepsilon} \text {. }
$$

Note that $\tilde{\phi}$ does not depend on the metric $K$. We may write $\theta=\phi+p$, where $p=\beta+\tau-q$. Note that $p$ is single valued and $|p| \leq r^{-1}|\log r|^{-1}$. Then $\bar{\theta}=\bar{\phi}+\bar{p}$, and hence

$$
|\bar{\theta}-\tilde{\phi}| \leq r^{-1}|\log r|^{-1}
$$

Recall that $\theta_{0}$ was the one-form which made the standard metric $K_{0}$ harmonic, and recall that it shares the same endomorphism $\phi$. In particular, by the same argument as above,

$$
\left|\bar{\theta}_{0}^{K_{0}}-\tilde{\phi}\right| \leq r^{-1}|\log r|^{-1}
$$

This proves the lemma. 
We will improve Lemma 7.2:

Lemma 7.4. $|2 \theta-\psi| \leq r^{-1}|\log r|^{-1}$.

Proof. The idea is to make $\phi$ into a $d^{\prime \prime}$-holomorphic endomorphism $\varphi$, and then to compare $\varphi$ with $\psi$. We first claim that $|[\bar{\theta}, \phi]| \leq r^{-2+\varepsilon}$. To see this, write $\theta=\alpha+\tau^{0}+\beta+\tau^{+}$. Recall that $\bar{\tau}^{0}$ commutes with $\alpha$ (as does $\bar{\alpha}$ ). On the other hand, $\left|\beta+\tau^{+}\right| \leq r^{-1+\varepsilon}$. Also, $\phi=\alpha+q$ and $|q| \leq r^{-1+\varepsilon}$. Putting these together gives the claim (it works uniformly on any sector, but the claim is single valued).

We may now use the operator $H$ which solves the $d^{\prime \prime}$-problem, discussed in Lemma 7.1. The above claim implies (after decreasing $\varepsilon$ ) that

$$
|H([\bar{\theta}, \phi])| \leq r^{-1+\varepsilon}
$$

Set $\varphi=\phi-H([\bar{\theta}, \phi])$. Note that $d^{\prime \prime}(\phi)=\bar{\partial}(\phi)+\bar{\theta}(\phi)=[\bar{\theta}, \phi]$. Thus we have $d^{\prime \prime}(\varphi)=0$. On the other hand, $|\varphi-\phi| \leq r^{-1+\varepsilon}$, so

$$
|\varphi-\theta| \leq r^{-1}|\log r|^{-1} \text {. }
$$

Now, $2 \varphi-\psi$ is a $d^{\prime \prime}$-holomorphic endomorphism of $V$, so we have $|2 \varphi-\psi| \sim$ $r^{\alpha}|\log r|^{k}$ for some $\alpha$ and $k$. We have

$$
|2 \varphi-\psi| \leq r^{-1}|\log r|^{-1}+|2 \theta-\psi|,
$$

so by Lemma 7.2 ,

$$
\int|2 \varphi-\psi|^{2}|\log r|^{1-\varepsilon} d \mathrm{vol}<\infty
$$

From this we conclude that $\alpha \geq-1$, and if $\alpha=-1$ then $k \leq-1$. Thus $|2 \varphi-\psi| \leq r^{-1}|\log r|^{-1}$, which implies the lemma.

Corollary 7.5. We have $\left|\psi-\psi_{0}\right| \leq r^{-1}|\log r|^{-1}$.

Proof. The same lemma as above holds for the system $V_{0}$, namely $\left|2 \theta_{0}-\psi_{0}\right|$ $\leq r^{-1}|\log r|^{-1}$. On the other hand, recall that $\left|\theta_{0}-\theta\right| \leq r^{-1+\varepsilon}$. Therefore $\left|2 \theta-\psi_{0}\right| \leq r^{-1}|\log r|^{-1}$. Putting this together with the conclusion of the above lemma, we get the corollary.

Consider the identity map $I$ of $E$ thought of as a map from $V_{0}$ to $V$. Since the metrics of $V_{0}$ and $V$ are mutually bounded, $I$ is bounded. Choose adapted bases $v_{i}^{0}$ and $v_{j}, d_{0}^{\prime \prime}$ - or $d^{\prime \prime}$-holomorphic respectively. Say

$$
\left|v_{j}\right| \sim r^{\alpha_{j}}|\log r|^{k_{j}} \quad \text { and } \quad\left|v_{i}^{0}\right| \sim r^{\alpha_{i}^{0}}|\log r|^{k_{i}^{0}} \text {. }
$$

We may write

$$
I=\sum_{i, j} I_{i j}\left(v_{i}^{0}\right)^{*} \otimes v_{j}
$$

We know that $\bar{\partial}(I)=0$. Therefore

$$
d^{\prime \prime}(I)=\bar{\theta}_{K_{0} K}(I)=\bar{\theta}_{K}-\bar{\theta}_{0, K_{0}} \stackrel{\text { def }}{=} M .
$$


On the other hand, $d^{\prime \prime}(I)=\sum_{i, j} \bar{\partial}\left(I_{i j}\right)\left(v_{i}^{0}\right)^{*} \otimes v_{j}$. Thus $\bar{\partial}\left(I_{i j}\right)=m_{i j}$. Set $\alpha_{i j}=\alpha_{j}-\alpha_{i}^{0}$ and $k_{i j}=k_{j}-k_{i}^{0}$. Then $\left(v_{i}^{0}\right) \otimes v_{j}$ is an adapted basis for $\operatorname{Hom}\left(V_{0}, V\right)$ with norms $r^{\alpha_{i j}}|\log r|^{k_{i j}}$ respectively. In particular, we have $\left|I_{i j}\right| \leq C r^{-\alpha_{i j}}|\log r|^{-k_{i j}}$. We may assume that the $\left(\alpha_{i j}, k_{i j}\right)$ are arranged in the interval from $(-2,-1)$ to $(-1,-1)$ inclusive (referring to the obvious lexicographic order).

Let $\lambda_{i}^{0}$ and $\lambda_{j}$ denote the residues of the eigenvalues of $\psi^{0}$ and $\psi$ for the vectors $v_{i}^{0}$ and $v_{j}$ (we may assume that they are eigenvectors).

Proposition 7.6. There is a sequence of points $x_{n} \rightarrow 0$ and constants $\varepsilon_{n} \rightarrow 0$ such that for any $i, j$ with $\left(\lambda_{i}^{0}, \alpha_{i}^{0}(\bmod \mathbf{Z}), k_{i}^{0}\right) \neq\left(\lambda_{j}, \alpha_{j}(\bmod \mathbf{Z}), k_{j}\right)$, then $\left.\left|I_{i j} r^{\alpha_{i j}}\right| \log r\right|^{k_{i j}} \mid\left(x_{n}\right) \leq \varepsilon_{n}$.

As a corollary of this proposition we get the following, which is the main theorem of the section.

Theorem 7. The dimensions of the spaces of vectors with a given $(\lambda, \alpha(\bmod \mathbf{Z})$, $k$ ) are the same for $V_{0}$ and $V$. In particular, the associated graded pieces and residues are the same.

Proof. There is an $\varepsilon$ such that if a matrix is divided into blocks, and all entries outside the blocks are of size less than $\varepsilon$, and all other entries are bounded and the determinant is one, then the blocks must be square. To prove this, it suffices to consider the case of two blocks. If they are not square, then this means that there is an $i$ such that the $(i, i)$ entry is not in one of the blocks, and such that the entries $(j, k)$ for $j \geq i$ and $k \leq i$ are not in the blocks (or the reverse, which is the same). Now $\operatorname{det}\left(a_{j k}\right)=\sum_{\sigma} \pm a_{1 \sigma(1)} \cdots a_{n \sigma(n)}$. But for every permutation $\sigma$ there is a $j \geq i$ such that $\sigma(j) \leq i$ (since you cannot fit the $n+1-i$ numbers $j \geq i$ into the interval $k>i$ which has length $n-i)$. Thus there is one $j$ such that $\left|a_{j \sigma(j)}\right| \leq \varepsilon$. In particular, the determinant is less than $\varepsilon C^{n-1} n$ !. If $\varepsilon$ is small then this cannot be equal to 1 .

Now apply this fact to the matrix whose entries are $I_{i j} r^{\alpha_{i j}}|\log r|^{k_{i j}}$. The determinant of this matrix is 1 (since $\left.\operatorname{det}\left(V_{0}\right)=\operatorname{det}(V)\right)$. If we arrange the basis vectors according to the values of $(\lambda, \alpha(\bmod Z), k)$ then we get exactly a block decomposition as described above. The previous paragraph implies that the blocks are square, which is the statement of the theorem.

Proof of Proposition 7.6. The rest of the section will be occupied with the proof of the proposition. Define the following fancy norms:

$$
\begin{gathered}
\|f\|_{Z}^{2}=\int|f|^{2} \frac{d \text { vol }}{r^{2}|\log r|}, \\
\|f\|_{W}=\int|f|^{\frac{d \operatorname{vol}}{r^{2}|\log r| \log |\log r|}} .
\end{gathered}
$$


Notice the following fact: if $\|f\|_{W}<\infty$ then there is a sequence of points $x_{n} \rightarrow 0$ and constants $\varepsilon_{n} \rightarrow 0$ such that $\left|f\left(x_{n}\right)\right| \leq \varepsilon_{n}$ (and the same is true for the $Z$ norm). Furthermore, if $\left\|f_{i}\right\|_{W}<\infty$ for finitely many $f_{i}$ then we may choose a sequence $\left\{x_{n}\right\}$ which works for all of the $f_{i}$. This is because

$$
\int \frac{d \text { vol }}{r^{2}|\log r| \log |\log r|}=\infty \text {. }
$$

Thus to prove the proposition it suffices to show under the same hypotheses that $\left\|I_{i j} r^{\alpha_{i j}}|\log r|^{k_{i j}}\right\|_{W}<\infty$.

Lemma 7.7. We have $|M| \leq r^{-1}|\log r|^{-1}$ and, in fact, the stronger estimate $\|M r \log r\|_{Z}<\infty$.

Proof. The first part is Lemma 7.3. Since $D^{\prime \prime}(I)=0$ we have the Weitzenböck formula

$$
\Delta|I|^{2} \leq-\left|D^{\prime}(I)\right|^{2} .
$$

This estimate holds weakly across the puncture. Furthermore $|I|^{2}$ is bounded. Therefore, since $|\log r|$ is the Greens function for the Laplacian,

$$
\int\left|D^{\prime}(I)\right|^{2}|\log r| d \mathrm{vol}<\infty .
$$

Note that $M$ is the $(0,1)$ component of $D^{\prime}(I)$, so this gives the desired inequality.

As a consequence of this lemma, we have $\left|m_{i j}\right| \leq r^{-\alpha_{i j}-1}|\log r|^{-k_{i j}-1}$ and $\left\|m_{i j} r^{\alpha_{i j}+1}|\log r|^{k_{i j}+1}\right\|_{Z}<\infty$. From the first estimates, and the result used in Lemma 7.1, we have, whenever $\alpha_{i j}$ is not an integer,

$$
\left|h\left(m_{i j}\right)\right| \leq r^{-\alpha_{i j}}|\log r|^{-k_{i j}-1} .
$$

In particular, $\left\|h\left(m_{i j}\right) r^{\alpha_{i j}}|\log r|^{k_{i j}}\right\|_{W}<\infty$. Suppose $\alpha_{i j}$ is an integer. We may also treat the case $k_{i j} \neq 0$ with the following lemma.

Lemma 7.8. Suppose $f$ is a $(0,1)$-form with $\left\|f r|\log r|^{-k}\right\|_{Z}<\infty$ and $|f| \leq$ $r^{-1}|\log r|^{k}$, with $k>-1$. Then $\left\|h(f)|\log r|^{-k-1}\right\|_{W}<\infty$. Similarly, if $\left\|f r^{2}|\log r|^{-k}\right\|_{Z}<\infty$ and $|f| \leq r^{-2}|\log r|^{k}$ with $k<-1$, then

$$
\left\|h(f) r|\log r|^{-k-1}\right\|_{W}<\infty .
$$

Proof. Note that we start with an estimate for \|\|$_{Z}$ and obtain an estimate for \|\|$_{W}$. We proceed as in the proof of Lemma 7.2. Let

$$
I(x)=\int_{|y| \leq B} \frac{|f(y)|}{|x-y|} d \operatorname{vol}(y) .
$$

Using the same regions as before, and following similar arguments, we get

$$
I(x) \leq I_{(\mathrm{i})}(|x|)+I_{(\mathrm{ii})}(x)+I_{(\mathrm{iii})}(|x|),
$$


where

$$
I_{(\mathrm{i})}(r)=\frac{1}{r} \int_{|y|=0}^{r}|f(y)| d \operatorname{vol}(y)
$$

and

$$
I_{(\mathrm{iii})}(r)=\int_{|y|=r}^{B} \frac{|f(y)|}{|y|} d \operatorname{vol}(y) .
$$

The middle integral $I_{(\mathrm{ii})}(x)$ is bounded by $|\log r|^{k}$ in the first case, and by $r^{-1}|\log r|^{k}$ in the second case, so it poses no problem and we can ignore it. Similarly, in the first case the first integral $I_{(\mathrm{i})}(r)$ is bounded by $|\log r|^{k}$ and in the second case the integral $I_{(\mathrm{iii})}(r)$ is bounded by $r^{-1}|\log r|^{k}$, so we may ignore them.

Go now to the first case. We know that

$$
\int|f|^{2} \frac{d \text { vol }}{|\log r|^{2 k+1}}<\infty
$$

and we have to show that

$$
\int I_{(\mathrm{iii})}(r) \frac{r d r}{r^{2}|\log r|^{k+2} \log |\log r|}<\infty .
$$

We have $I_{(\mathrm{iii})}(r)=\int_{r}^{B}\left|f\left(u e^{i \theta}\right)\right| d u$ where the integration over the angle $\theta$ is to be understood from now on. Thus our integral in question is

$$
\begin{aligned}
\int_{r=0}^{B} & \int_{u=r}^{B}\left|f\left(u e^{i \theta}\right)\right| \frac{d u d r}{r|\log r|^{k+2} \log |\log r|} \\
& =\int_{u=0}^{B}\left|f\left(u e^{i \theta}\right)\right| d u \int_{r=0}^{u} \frac{d r}{r|\log r|^{k+2} \log |\log r|} \\
& \leq C \int_{u=0}^{B}\left|f\left(u e^{i \theta}\right)\right| \frac{d u}{|\log u|^{k+1} \log |\log u|} .
\end{aligned}
$$

The last step is due to the fact that $-k-2<-1$. Now apply Hölder's inequality:

$$
\begin{aligned}
& \int_{u=0}^{B}\left|f\left(u e^{i \theta}\right)\right| \frac{d u}{|\log u|^{k+1} \log |\log u|} \\
& \quad \leq\left(\int \frac{|f|^{2} u d u}{|\log u|^{2 k+1}}\right)^{1 / 2}\left(\int \frac{d u}{u|\log u|(\log |\log u|)^{2}}\right)^{1 / 2} .
\end{aligned}
$$

The first term on the right is finite by assumption, and the second term on the right is clearly finite, so our integral is finite. This proves the first part of the lemma.

For the second part, we know that

$$
\int|f|^{2} \frac{r^{2} d \text { vol }}{|\log r|^{2 k+1}}<\infty
$$


and we have to show that

$$
\int r I_{(\mathrm{i})}(r) \frac{r d r}{r^{2}|\log r|^{k+2} \log |\log r|}<\infty .
$$

We have $r I_{(\mathrm{i})}(r)=\int_{0}^{r}\left|f\left(u e^{i \theta}\right)\right| u d u$. Thus the integral in question is

$$
\begin{aligned}
\int_{r=0}^{B} & \int_{u=0}^{r}\left|f\left(u e^{i \theta}\right)\right| \frac{u d u d r}{r|\log r|^{k+2} \log |\log r|} \\
& =\int_{u=0}^{B}\left|f\left(u e^{i \theta}\right)\right| u d u \int_{r=u}^{B} \frac{d r}{r|\log r|^{k+2} \log |\log r|} \\
& \leq C \int_{u=0}^{B}\left|f\left(u e^{i \theta}\right)\right| \frac{u d u}{|\log u|^{k+1} \log |\log u|} .
\end{aligned}
$$

The last step is due to the fact that $-k-2>-1$. Now apply Hölder's inequality:

$$
\begin{aligned}
& \int_{u=0}^{B}\left|f\left(u e^{i \theta}\right)\right| \frac{u d u}{|\log u|^{k+1}|\log | \log u \mid} \\
& \quad \leq\left(\int \frac{|f|^{2} u^{3} d u}{|\log u|^{2 k+1}}\right)^{1 / 2}\left(\int \frac{d u}{u|\log u|(\log |\log u|)^{2}}\right)^{1 / 2} .
\end{aligned}
$$

The first term on the right is again finite by assumption, and the second term on the right is finite, so our integral is finite. This completes the proof of the lemma.

Corollary 7.9. If $\alpha_{i j}$ is not an integer or if it is an integer but $k_{i j} \neq 0$, then

$$
\left\|h\left(m_{i j}\right) r^{\alpha_{i j}}|\log r|^{k_{i j}}\right\|_{W}<\infty \text {. }
$$

Proof. This follows from our estimate for $m_{i j}$ and from the lemma.

Corollary 7.10. If $\alpha_{i j}$ is not an integer or if it is an integer but $k_{i j} \neq 0$, then

$$
\left\|I_{i j} r^{\alpha_{i j}}|\log r|^{k_{i j}}\right\|_{W}<\infty \text {. }
$$

Proof. $I_{i j}-h\left(m_{i j}\right)$ are holomorphic functions, bounded by $r^{-\alpha_{i j}}|\log r|^{-k_{i j}}$. If $\alpha_{i j}$ is not an integer or if $k_{i j} \neq 0$ then these holomorphic functions are in fact bounded by $r^{-\alpha_{i j}}|\log r|^{-k_{i j}-1 / 2}$. Thus these holomorphic functions satisfy the same bounds desired for $I_{i j}$. On the other hand, the previous corollary gives these bounds for the $h\left(m_{i j}\right)$. Adding the two, we get the corollary.

Lemma 7.11. If $\lambda_{i}^{0} \neq \lambda_{j}$, then $\left|I_{i j}\right| \leq r^{-\alpha_{i j}}|\log r|^{-k_{i j}-1}$

Proof. Corollary 7.5 says that $\left|\psi_{0}-\psi\right| \leq r^{-1}|\log r|^{-1}$. Note that

$$
\psi=\sum \lambda_{j} \frac{d z}{z}\left(v_{j}\right)^{*} \otimes v_{j}
$$

and similarly for $\psi_{0}$. Therefore

$$
\psi_{0}-\psi=\sum_{i, j}\left(\lambda_{i}^{0}-\lambda_{j}\right) \frac{d z}{z} I_{i j}\left(v_{i}^{0}\right)^{*} \otimes v_{j} .
$$


The adaptedness of our bases implies the result.

Note that this lemma gives the required estimate in the case $\lambda_{i}^{0} \neq \lambda_{j}$. Together, the above arguments prove the proposition. This also completes the proof of the main theorem.

Remark. If the eigenvalues of $\theta$ are purely imaginary, so the corresponding local system has trivial filtration, then you can easily tell the conjugacy classes of the monodromy transformations from the filtered regular Higgs bundle $E$, more specifically from the decomposition of $\operatorname{Gr}(E, s)$ and the residue of $\theta$. The weight filtration for the unipotent part of the monodromy on any eigenspace is the same as the weight filtration of the residue of $\theta$ on the corresponding space, and the eigenvalues of the monodromy correspond to the eigenvalues of $\operatorname{res}(\theta)$ and the level $\alpha$ in the decomposition of $\operatorname{Gr}(E, s)$, as indicated in the table in $\S 5$.

\section{VARIATIONS OF HODGE STRUCTURE}

In this section we will describe which harmonic bundles come from complex variations of Hodge structure (see the Synopsis for definitions), in the same way as in the compact case. Namely, there is an action of $\mathbf{C}^{*}$ on the space of filtered regular Higgs bundles $t:(E, \theta) \mapsto(E, t \theta)$, which preserves stability and degree, and we prove the following.

Theorem 8. The set of harmonic bundles which come from complex variations of Hodge structure is equal to the set of those which are fixed by the action of $\mathbf{C}^{*}$ (or any infinite subgroup thereof).

Proof. A filtered regular Higgs bundle $E$ which is decomposed, $E=\bigoplus E^{p}$ with $\theta: E^{p} \rightarrow E^{p-1} \otimes \Omega_{X}^{1}$, is a system of Hodge bundles. In a preliminary version of the present work, contained in the last section of [19], the stable filtered regular systems of Hodge bundles were seen to correspond to complex variations of Hodge structures.

We will show that if $(E, \theta)$ is a filtered regular Higgs bundle, and $(E, \theta) \cong$ $(E, t \theta)$, then $(E, \theta)$ comes from a filtered regular system of Hodge bundles. Suppose $t$ is a nonzero complex number, not of finite order. Let $\varphi: E \rightarrow E$ be the given isomorphism intertwining $\theta$ with $t \theta$. Decompose $E$ into eigenspaces for $\varphi$, and label them according to powers of $t$, so $E=\bigoplus E^{p}$ and $\theta: E^{p} \rightarrow$ $E^{p-1} \otimes \Omega_{X}^{1}$. The only point of difference with the compact case is that we must show that the decomposition of $E$ is compatible with the filtration, in other words that $E_{\alpha}=\bigoplus E_{\alpha}^{p}$. But each of the bundles $E_{\alpha}$ is preserved by $\varphi$ (since $\varphi$ is a morphism of filtered bundles), so $E_{\alpha}$ has an eigenspace decomposition. This decomposition is determined by what happens at the generic point of $X$, so the eigenspace in $E_{\alpha}$ is the intersection of the eigenspace in $j_{*} E$ with $E_{\alpha}$. Thus we get a filtered regular system of Hodge bundles. To complete the proof from here, see [19]. 
Remark. When checking stability of a filtered regular system of Hodge bundles, it suffices to consider only subsystems of Hodge bundles [19].

Corollary 8.1. Suppose a semisimple representation of the fundamental group of $X$ has monodromy transformations at the punctures whose eigenvalues have norm 1 , and suppose that there is only one representation with those specified monodromy transformations. Then the representation comes from a complex variation of Hodge structure.

Proof. Look at the harmonic bundle associated to the semisimple representation (with trivial filtrations of the local system). The condition that the eigenvalues of the monodromy transformations have norm one means that the residue of $\theta$ is nilpotent. Thus the isomorphism class of $\operatorname{res}(\theta)$ is preserved by the action of $\mathrm{C}^{*}$. By the main theorem of the previous section, this means that the isomorphism classes of the monodromy transformations are preserved by the action of $\mathbf{C}^{*}$. The other hypothesis now implies that the representation is preserved by $\mathbf{C}^{*}$, so it comes from a variation of Hodge structure.

Remark. If, as is to be expected, the correspondence we have developed has any reasonable continuity properties, then we may replace the strong rigidity hypothesis in the corollary by the weaker hypothesis of local rigidity, i.e. that the representation has no deformations which preserve the monodromy transformations at the punctures.

\section{REFERENCES}

1. L. V. Ahlfors, An extension of Schwarz's lemma, Trans. Amer. Math. Soc. 43 (1938), 359364.

2. K. Corlette, Flat G-bundles with canonical metrics, J. Differential Geom. 28 (1988), 361382.

3. M. Cornalba and P. Griffiths, Analytic cycles and vector bundles on noncompact algebraic varieties, Invent. Math. 28 (1975), 1-106.

4. P. Deligne, Equations differentielles à points singuliers reguliers, Lecture Notes in Math., vol. 163, Springer, 1970.

5. S. K. Donaldson, Anti self dual Yang-Mills connections over complex algebraic surfaces and stable vector bundles, Proc. London Math. Soc. (3) 50 (1985), 1-26.

6. Infinite determinants, stable bundles, and curvature, Duke Math. J. 54 (1987), 231247.

7. _ Twisted harmonic maps and self-duality equations, Proc. London Math. Soc. (3) 55 (1987), 127-131.

8. J. Eells and J. H. Sampson, Harmonic mappings of Riemannian manifolds, Amer. J. Math. 86 (1964), 109-160.

9. P. Griffiths, Periods of integrals on algebraic manifolds. I, II, Amer. J. Math. 90 (1968); III, Inst. Hautes Études Sci. Publ. Math. 38 (1970).

10. P. Griffiths et al., Topics in transcendental algebraic geometry, Princeton Univ. Press, Princeton, NJ, 1984.

11. P. Griffiths and W. Schmid, Locally homogeneous complex manifolds, Acta Math. 123 (1969), 253-302.

12. R. S. Hamilton, Harmonic maps of manifolds with boundary, Lecture Notes in Math., vol. 471, Springer-Verlag, Berlin and New York, 1975. 
13. N. J. Hitchin, The self-duality equations on a Riemann surface, Proc. London Math. Soc. (3) 55 (1987), 59-126.

14. M. Kashiwara, Vanishing cycles and holonomic systems of differential equations, Lecture Notes in Math., vol. 1016, Springer-Verlag, Berlin and New York, 1983, pp. 134-142.

15. B. Malgrange, Polynôme de Bernstein-Sato et cohomologie évanescente, Astérisque 101-102 (1983), 243-267.

16. V. B. Mehta and C. S. Seshadri, Moduli of vector bundles on curves with parabolic structures, Math. Ann. 248 (1980), 205-239.

17. M. S. Narasimhan and C. S. Seshadri, Stable and unitary bundles on a compact Riemann surface, Ann. of Math. (2) 82 (1965), 540-564.

18. W. Schmid, Variation of Hodge structure: the singularities of the period mapping, Invent. Math. 22 (1973), 211-319.

19. C. T. Simpson, Constructing variations of Hodge structure using Yang-Mills theory and applications to uniformization, J. Amer. Math. Soc. 1 (1988), 867-918.

20. __ Higgs bundles and local systems, preprint, Princeton Univ., 1989.

21. K. K Uhlenbeck and S. T. Yau, On the existence of Hermitian-Yang-Mills connections in stable vector bundles, Comm. Pure Appl. Math. 39 (1986), 257-293.

22. S. Zucker, Hodge theory with degenerating coefficients: $L^{2}$ cohomology in the Poincaré metric, Ann. of Math. (2) 109 (1979), 415-476.

23. H. Esnault and E. Viehweg, Logarithmic de Rham complexes and vanishing theorems, Invent. Math. 86 (1986), 161-194.

Department of Mathematics, Princeton University, Princeton, New Jersey 08544 